Pacific

Journal of

Mathematics

A SCHLÄFLI DIFFERENTIAL FORMULA FOR SIMPLICES IN SEMI-RIEMANNIAN HYPERQUADRICS, GAUSS-BONNET FORMULAS FOR SIMPLICES IN THE DE SITTER SPHERE AND THE DUAL VOLUME OF A HYPERBOLIC SIMPLEX

Eva SuÁREZ-Peiró 


\title{
A SCHLÄFLI DIFFERENTIAL FORMULA FOR SIMPLICES IN SEMI-RIEMANNIAN HYPERQUADRICS, GAUSS-BONNET FORMULAS FOR SIMPLICES IN THE DE SITTER SPHERE AND THE DUAL VOLUME OF A HYPERBOLIC SIMPLEX
}

\author{
Eva SuÁREZ-PEIRó
}

\begin{abstract}
In this paper we prove a Schläfli differential formula for the volume of simplices in central unit hyperquadrics of semiEuclidean space $\mathbb{R}_{q}^{n+1}$. Then we apply this result to obtain Gauss-Bonnet formulas for simplices with riemannian faces in the de Sitter sphere, and to generalize a formula of L. Santaló relating the volume of a hyperbolic simplex with the measure of the set of hyperbolic hyperplanes intersecting it.
\end{abstract}

\section{Introduction.}

Schläfli's differential formula plays a central role in the computation of the volume of hyperbolic and spherical polyhedra of any dimension. Given a family of $n$-simplices $\Delta$ which vary in a differentiable manner in a nonEuclidean space of dimension $n$ and constant curvature $\kappa=1$ or $\kappa=-1$, the Schläfli formula expresses the differential of the volume of $\Delta$ in terms of the volumes of its faces of codimension 2 and the dihedral angles at these faces. Namely,

$$
d \mathrm{~V}_{n}(\Delta)=\frac{\kappa}{n-1} \sum_{F} \mathrm{~V}_{n-2}(F) d \alpha_{F}
$$

where the sum is taken over all faces $F$ of codimension 2 of $\Delta, \mathrm{V}_{n-2}(F)$ denotes the $((n-2)$-dimensional $)$ volume of face $F$ and $\alpha_{F}$ denotes the dihedral angle at face $F$.

Around 1852, L. Schläfli proved this formula for spherical simplices of any dimension ([Schlä]). In $1936 \mathrm{H}$. Kneser gave a different proof, which he could easily generalize to the hyperbolic case ([Kne]). A more recent, very recommended reference is J. Milnor's paper ([Mil]), where some remarks on the history of the Schläfli differential formula can be found, as well as a new proof which is particularly transparent. In the last years great use has been made of this formula to compute the volume of hyperbolic 3-manifolds, possibly with cone-type singularities (cf. $\left[\mathbf{H L M}_{1}\right]$, $\left[\mathbf{H L M}_{2}\right],\left[\mathbf{H L M}_{3}\right]$ ), following an idea of C. Hodgson ([Ho]). It has also been used to study the 
volume of hyperbolic spaceforms in general (cf. [Kel]). Recently F. Bonahon has found a Schläfli type formula for the volume of the convex core of hyperbolic 3-manifolds (cf. [Bo]).

On the other hand, it is also interesting to compute the volume of simplices contained in complete semi-riemannian manifolds of constant curvature; some work has been done in this direction by J.-M. Schlenker ([Schle 1$]$, $\left.\left[\mathbf{S c h l e}_{2}\right]\right)$. An important example is the $n$-dimensional de Sitter sphere $\mathbb{S}_{1}^{n}$, which is a complete Lorentz manifold of constant curvature 1 . There is a duality relationship between $\mathbb{S}_{1}^{n}$ and $n$-dimensional hyperbolic space $\mathbb{H}^{n}$ (when both are considered as submanifolds of Minkowski space $\mathbb{R}_{1}^{n+1}$ ), associating to each point $\mathbf{v}$ in $\mathbb{S}_{1}^{n}$ the hyperplane in $\mathbb{H}^{n}$ with unit normal $\mathbf{v}$ (cf. $[\mathbf{H R}]$ ). Therefore, a Schläfli formula for the volume of simplices in $\mathbb{S}_{1}^{n}$ can be used to relate the volume of a hyperbolic simplex with the measure of the set of hyperplanes intersecting it. (We will call the measure of this set of hyperplanes, the dual volume of the given hyperbolic simplex.) For $n=3$

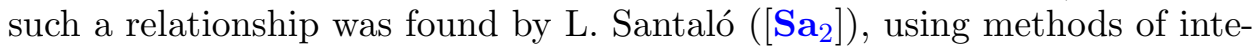
gral geometry. (An analogous equation, relating the volume of a spherical tetrahedron with the measure of the set of planes intersecting it, was proved by Milnor using the spherical Schläfli formula, cf. [Mil].) My PhD advisor, José María Montesinos ([Mo]), obtained a Schläfli differential equality for tetrahedra in $\mathbb{S}_{1}^{3}$ by differentiating the above mentioned formula of Santaló and then applying Schläfli's equation for hyperbolic tetrahedra. He suggested to me the possibility of obtaining a Schläfli formula for simplices in $\mathbb{S}_{1}^{n}$ following Kneser's proof, and of generalizing with it Santaló's equality to higher dimensions. This is, in a somewhat wider context, the aim of this paper, which is a part of my $\mathrm{PhD}$ thesis $([\mathbf{S u}])$.

In Section 1 we adapt and generalize Kneser's proof to obtain a Schläfli differential formula for a large class of simplices in any central unit hyperquadric of semi-Euclidean space $\mathbb{R}_{q}^{n+1}$ (cf. [O'N]). For this, a suitable definition of dihedral angle in a semi-riemannian geometry is necessary. This is a point that requires some care and it is tackled in subsection 1.4, where

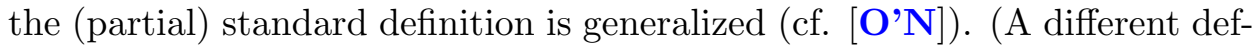
inition of dihedral angle (with complex values) in the semi-riemannian case, giving rise to a different version of the Schläfli formula, has been used by J.-M. Schlenker, cf. [Schle 1$]$, [Schle 2$]$.)

Then we give three applications of the Schläfli differential formula for simplices in the de Sitter sphere. In Section 2 we give a new proof of Santaló's formula in dimension 3 (relating the volume of a hyperbolic tetrahedron with the measure of the set of hyperplanes intersecting it), using the Schläfli formulas in $\mathbb{H}^{3}$ y $\mathbb{S}_{1}^{3}$. In this section we also introduce the notions of polar dual and complementary dual of a hyperbolic simplex of arbitrary dimension, which will be necessary to generalize Santaló's formula to higher dimensions. 
(The polar dual of a convex hyperbolic polyhedron has been extensively studied in $[\mathbf{R i}]$ and $[\mathbf{H R}]$.)

In Section 3 we obtain Gauss-Bonnet formulas for simplices in the $n$ dimensional de Sitter sphere $\mathbb{S}_{1}^{n}$ with riemannian faces. These formulas are analogous to the generalized Gauss-Bonnet formulas for spherical and hyperbolic simplices, which relate the volumes of all even- dimensional faces of the simplex and the dihedral angles at those faces (cf. [Sa 1$],[\mathbf{A V S}]$ ).

Finally Section 4 is a consequence of the previous two, and provides a generalization to higher dimensions of Santaló's formula relating the volume of a hyperbolic simplex with the measure of the set of hyperplanes intersecting it.

I thank José María Montesinos for suggesting the idea of this paper and for all his helpful advice and encouragement. I also thank Francis Bonahon for giving me the opportunity to talk about this result in a students' seminar at the I.H.P. in Paris, and I. Rivin and J.-M. Schlenker for their suggestions and for drawing my attention to their results.

Added in the revised version. After submission of this paper, I. Rivin and J.-M. Schlenker have obtained a smooth analogue of the Schläfli formula for the volume bounded by a hypersurface in a general Einstein manifold, using methods of differential geometry (cf. [RS]). The Schläfli formula for polyhedra in the de Sitter sphere proved in this paper follows as a corollary from their result. J-M. Schlenker and R. Souam have also obtained higher dimensional analogues of the Schläfli formula ([S-S]), which generalize the formulas given in Proposition 3.1. of this paper.

\section{A Schläfli differential formula for simplices in semi-riemannian hyperquadrics.}

\subsection{Background and definitions.}

To fix notations we recall now some standard definitions in semi-riemannian geometry (see $\left[\mathbf{O}^{\prime} \mathbf{N}\right]$ ).

Semi-Euclidean space $\mathbb{R}_{q}^{n+1}$ is $\mathbb{R}^{n+1}$ with the semi-riemannian metric defined by the bilinear form of index $q$

$$
\langle\mathbf{x}, \mathbf{y}\rangle=-\sum_{i=0}^{q-1} x_{i} y_{i}+\sum_{j=q}^{n} x_{j} y_{j}
$$

where $\mathbf{x}=\left(x_{0}, \ldots, x_{n}\right)$ and $\mathbf{y}=\left(y_{0}, \ldots, y_{n}\right)$. The norm of a vector $\mathbf{x} \in$ $\mathbb{R}_{q}^{n+1}$ is $|\mathbf{x}|=|\langle\mathbf{x}, \mathbf{x}\rangle|^{1 / 2}$. A vector $\mathbf{x}$ is timelike if $\langle\mathbf{x}, \mathbf{x}\rangle<0$, spacelike if $\langle\mathbf{x}, \mathbf{x}\rangle>0$ and null if $\langle\mathbf{x}, \mathbf{x}\rangle=0$.

Given $\epsilon \in\{ \pm 1\}$, the central unit hyperquadric of $\mathbb{R}_{q}^{n+1}$ with sign $\epsilon$ is the submanifold

$$
Q_{q}^{n}(\epsilon)=\left\{\mathbf{x} \in \mathbb{R}_{q}^{n+1} \mid\langle\mathbf{x}, \mathbf{x}\rangle=\epsilon\right\}
$$


It is a complete semi-riemannian $n$-dimensional manifold (not necessarily connected) with constant curvature $\epsilon$. The totally geodesic submanifolds of $Q_{q}^{n}(\epsilon)$ are the connected components of the intersection of $Q_{q}^{n}(\epsilon)$ with linear subspaces of $\mathbb{R}^{n+1}$. The isometries of $Q_{q}^{n}(\epsilon)$ are the restriction to $Q_{q}^{n}(\epsilon)$ of the linear automorphisms of $\mathbb{R}_{q}^{n+1}$ leaving $Q_{q}^{n}(\epsilon)$ invariant. Important examples are the $n$-dimensional sphere $\mathbb{S}^{n}=Q_{0}^{n}(1)$, the $n$-dimensional hyperbolic space $\mathbb{H}^{n}$, which is one of the two components of $Q_{1}^{n}(-1)$, the $n$-dimensional de Sitter sphere $\mathbb{S}_{1}^{n}=Q_{1}^{n}(1)$, and the 3-dimensional hyperquadric $Q_{2}^{3}(1)$, which can be identified with $S L(2, \mathbb{R})$ with the metric given by its canonical Killing form. From now on we will write $S_{q}^{n}(\epsilon)$ to denote any connected component of $Q_{q}^{n}(\epsilon)$, and we will also call $S_{q}^{n}(\epsilon)$ an $n$-dimensional hyperquadric.

Let us now define an $n$-simplex in the hyperquadric $S_{q}^{n}(\epsilon)$. We define a linear halfspace in $\mathbb{R}_{q}^{n+1}$ as a closed halfspace whose boundary is a linear hyperplane of $\mathbb{R}_{q}^{n+1}$. A simplicial cone in $\mathbb{R}_{q}^{n+1}$ is the intersection of $(n+1)$ linear halfspaces in general position (i.e., such that the $(n+1)$ boundary hyperplanes intersect only at the origin). A face of codimension $k$ of a simplicial cone $C(k=0, \ldots, n)$ is the intersection of $C$ with $k$ of the hyperplanes forming its boundary. Observe that the intersection of an arbitrary simplicial cone $C$ with the hyperquadric $S_{q}^{n}(\epsilon)$ can be empty, and it can also be non-compact.

Definition 1.1. An $n$-simplex in the $n$-dimensional hyperquadric $S_{q}^{n}(\epsilon)$ is the intersection of a simplicial cone with $S_{q}^{n}(\epsilon)$, when this intersection is nonempty and compact. A face of codimension $k$ of an $n$-simplex $\Delta \subset S_{q}^{n}(\epsilon)$ $(k=0, \ldots, n)$ is the intersection with $S_{q}^{n}(\epsilon)$ of a face of codimension $k$ of the corresponding simplicial cone.

Remark 1.1. Every simplicial cone $C$ in $\mathbb{R}_{q}^{n+1}$ can be written as

$$
C=\left\{x_{0} \mathbf{v}_{0}+\cdots+x_{n} \mathbf{v}_{n} \mid x_{0} \geq 0, \ldots, x_{n} \geq 0\right\}
$$

for some basis $\left\{\mathbf{v}_{0}, \ldots, \mathbf{v}_{n}\right\}$ of $\mathbb{R}_{q}^{n+1}$. We will use the following notation for the faces of codimension $k$ of a simplicial cone:

$$
C_{i_{1}} \ldots i_{k}=\left\{x_{0} \mathbf{v}_{0}+\cdots+x_{n} \mathbf{v}_{n} \mid x_{0} \geq 0, \ldots, x_{n} \geq 0 ; x_{i_{1}}=\cdots=x_{i_{k}}=0\right\} \text {. }
$$

A subset $\Delta \subset S_{q}^{n}(\epsilon)$ is an $n$-simplex if and only if it can be written as

$$
\Delta=\left\{x_{0} \mathbf{v}_{0}+\cdots+x_{n} \mathbf{v}_{n} \mid x_{0} \geq 0, \ldots, x_{n} \geq 0\right\} \cap S_{q}^{n}(\epsilon)
$$

for some basis $\left\{\mathbf{v}_{0}, \ldots, \mathbf{v}_{n}\right\}$ of $\mathbb{R}_{q}^{n+1}$ such that $\epsilon \cdot\left\langle x_{0} \mathbf{v}_{0}+\cdots+x_{n} \mathbf{v}_{n}, x_{0} \mathbf{v}_{0}+\right.$ $\left.\cdots+x_{n} \mathbf{v}_{n}\right\rangle>0$ if $x_{0} \geq 0, \ldots, x_{n} \geq 0$ and they are not all zero. We will denote the codimension $k$ faces of an $n$-simplex $\Delta$ as follows:

$$
\begin{aligned}
F_{i_{1} \ldots i_{k}}=\left\{x_{0} \mathbf{v}_{0}+\cdots+x_{n} \mathbf{v}_{n} \mid x_{0} \geq 0, \ldots, x_{n} \geq 0\right. \\
\left.x_{i_{1}}=\cdots=x_{i_{k}}=0\right\} \cap S_{q}^{n}(\epsilon) .
\end{aligned}
$$


Remark 1.2. Let $C$ be a simplicial cone in $\mathbb{R}_{q}^{n+1}$ such that $\Delta=C \cap S_{q}^{n}(\epsilon)$ is an $n$-simplex. Each codimension $k$ face $C_{i_{1}} \ldots i_{k}$ of $C$ spans an $(n+1-k)$ dimensional linear subspace $\left\langle C_{i_{1} \ldots i_{k}}\right\rangle$ of $\mathbb{R}_{q}^{n+1}$. If the induced metric in this subspace is non-degenerate of a certain index $\nu$, then $\left\langle C_{i_{1} \ldots i_{k}}\right\rangle \cap S_{q}^{n}(\epsilon)$ is an $(n-k)$-dimensional hyperquadric of type $S_{\nu}^{n-k}(\epsilon)$, and the corresponding face of $\Delta, F_{i_{1}} \ldots i_{k}=C_{i_{1} \ldots i_{k}} \cap S_{q}^{n}(\epsilon)$, is an $(n-k)$-simplex inside this hyperquadric. If the induced metric in $\left\langle C_{i_{1}} \ldots i_{k}\right\rangle$ is degenerate, then the face $F_{i_{1}} \ldots i_{k}$ of $\Delta$ also has a degenerate metric as a submanifold of $S_{q}^{n}(\epsilon)$.

In what follows we will restrict our attention to the class of $n$-simplices $\Delta$ in $S_{q}^{n}(\epsilon)$ satisfying the following additional condition $(*)$, in order to avoid dividing by zero in the coming calculations:

(*) All faces of codimension 1 and 2 of $\Delta$ have a non-degenerate metric.

\subsection{The volume function.}

Let $\Delta$ be an $n$-simplex in $S_{q}^{n}(\epsilon)$, and let $C$ be the simplicial cone of $\mathbb{R}_{q}^{n+1}$ such that $\Delta=C \cap S_{q}^{n}(\epsilon)$. To compute the volume of $\Delta$, it is very useful to consider the following idea of Kneser ([Kne $]$ ). Define the function $r(\mathbf{x})=\sqrt{|\langle\mathbf{x}, \mathbf{x}\rangle|}$, for $\mathbf{x} \in \mathbb{R}_{q}^{n+1}$. Then the following relation holds inside the simplicial cone $C$ :

$$
d \mathbb{R}_{q}^{n+1}=r^{n} d r \wedge d S_{q}^{n}(\epsilon)
$$

where $d \mathbb{R}_{q}^{n+1}$ denotes the volume form of $\mathbb{R}_{q}^{n+1}$ (with the standard orientation) and $d S_{q}^{n}(\epsilon)$ denotes the volume form of $S_{q}^{n}(\epsilon)$ (oriented in such a way that the normal vector $\frac{\partial}{\partial r}$ followed by a positive basis of tangent vectors to $S_{q}^{n}(\epsilon)$, form a positive basis of $\left.\mathbb{R}_{q}^{n+1}\right)$.

Hence the volume $V_{n}(\Delta)$ of $\Delta$ can be obtained from the following equality:

$$
\begin{aligned}
\int_{C} e^{-\frac{r^{2}}{2}} d \mathbb{R}_{q}^{n+1} & =\int_{C} r^{n} e^{-\frac{r^{2}}{2}} d r \wedge d S_{q}^{n}(\epsilon) \\
& =\int_{\Delta} d S_{q}^{n}(\epsilon) \cdot \int_{0}^{+\infty} r^{n} e^{-\frac{r^{2}}{2}} d r \\
& =2^{\frac{n-1}{2}} \Gamma\left(\frac{n+1}{2}\right) \mathrm{V}_{\mathrm{n}}(\Delta) .
\end{aligned}
$$

Let $\left\{\mathbf{v}_{0}, \ldots, \mathbf{v}_{n}\right\}$ be a positive basis of $\mathbb{R}_{q}^{n+1}$ such that $C=\left\{x_{0} \mathbf{v}_{0}+\cdots+\right.$ $\left.x_{n} \mathbf{v}_{n} \mid x_{0} \geq 0, \ldots, x_{n} \geq 0\right\}$. Denote by $\left(x_{0}, \ldots, x_{n}\right)$ the coordinates in this basis and let $\Phi\left(x_{0}, \ldots, x_{n}\right)=\left\langle x_{0} \mathbf{v}_{0}+\cdots+x_{n} \mathbf{v}_{n}, x_{0} \mathbf{v}_{0}+\cdots+x_{n} \mathbf{v}_{n}\right\rangle$ be the quadratic form of $\mathbf{R}_{q}^{n+1}$. Then inside the simplicial cone $C$ we have $\Phi=\epsilon \cdot r^{2}$. On the other hand, consider the parallelepiped determined in $\mathbb{R}_{q}^{n+1}$ by the vectors $\mathbf{v}_{0}, \ldots, \mathbf{v}_{n}$, and denote by $\mathrm{V}\left(\mathbf{v}_{0}, \ldots, \mathbf{v}_{n}\right)$ its volume in 
$\mathbb{R}_{q}^{n+1}$ (which coincides with its euclidean volume). Then the volume form of $\mathbb{R}_{q}^{n+1}$ is

$$
d \mathbb{R}_{q}^{n+1}=\mathrm{V}\left(\mathbf{v}_{0}, \ldots, \mathbf{v}_{n}\right) d x_{0} \wedge \ldots \wedge d x_{n} .
$$

Therefore, Equation (1) can be written in the coordinates $\left(x_{0}, \ldots, x_{n}\right)$ as

$$
2^{\frac{n-1}{2}} \Gamma\left(\frac{n+1}{2}\right) \mathrm{V}_{\mathrm{n}}(\Delta)=\mathrm{V}\left(\mathbf{v}_{0}, \ldots, \mathbf{v}_{n}\right) \cdot \int_{C} e^{-\epsilon \Phi / 2} d x_{0} \ldots d x_{n} .
$$

\subsection{The volume differential.}

The space $\mathcal{S}$ of all $n$-simplices in $S_{q}^{n}(\epsilon)$ has a natural structure of $n(n+1)$ dimensional manifold, with charts consisting, for instance, of products of sufficiently small neighborhoods of the $n+1$ vertices inside $S_{q}^{n}(\epsilon)$. The volume function $V_{n}: \mathcal{S} \rightarrow \mathbb{R}$, associating to each simplex $\Delta$ its volume $V_{n}(\Delta)$, is a differentiable map, and we are interested in finding a formula for its differential.

To do this, we will look at an arbitrary point $\Delta \in \mathcal{S}$ and consider a special basis formed by $n(n+1)$ linearly independent tangent vectors of $\mathcal{S}$ at $\Delta$, and we will compute the value of the form $d \mathrm{~V}_{n}$ applied to each of these tangent vectors. This special basis is defined as follows: for each of the $n+1$ vertices of $\Delta$, we take $n$ linearly independent tangent vectors of $\mathcal{S}$ at $\Delta$ that represent $n$ ways of moving the chosen vertex in different directions, keeping all the other vertices fixed. More explicitly, the chosen vertex will move along the line joining it to each of the $n$ remaining vertices, as described below.

Let $C=\left\{x_{0} \mathbf{v}_{0}+\cdots+x_{n} \mathbf{v}_{n} \mid x_{0} \geq 0, \ldots, x_{n} \geq 0\right\}$ be the simplicial cone such that $\Delta=C \cap S_{q}^{n}(\epsilon)$. Since the ordering of the vertices is arbitrary, it is enough to study the case where the vertex $\mathbf{v}_{1}$ moves towards the vertex $\mathbf{v}_{0}$ and all other vertices remain fixed. For every $t \in \mathbb{R}$ consider the basis formed by the vectors $\left\{\begin{array}{l}\mathbf{v}_{0}(t)=\mathbf{v}_{0} \\ \mathbf{v}_{1}(t)=\mathbf{v}_{1}-t \mathbf{v}_{0} \\ \mathbf{v}_{i}(t)=\mathbf{v}_{i} \text { if } 2 \leq i \leq n\end{array}\right.$. When $t$ is sufficiently close to 0 , the simplicial cone $C_{t}=\left\{\lambda_{0} \mathbf{v}_{0}(t)+\cdots+\lambda_{n} \mathbf{v}_{n}(t) \mid \lambda_{0} \geq 0, \ldots, \lambda_{n} \geq 0\right\}$ is such that $\Delta_{t}=C_{t} \cap S_{q}^{n}(\epsilon)$ is an $n$-simplex. Then we will take as one of the tangent vectors of $\mathcal{S}$ at $\Delta$, the tangent vector to the path $\Delta_{t}$ at time $t=0$.

The value of the form $d \mathrm{~V}_{n}$ applied to this tangent vector is $\left.\frac{d \mathrm{~V}_{n}\left(\Delta_{t}\right)}{d t}\right|_{t=0}$. Using formula (2),

$$
2^{\frac{n-1}{2}} \Gamma\left(\frac{n+1}{2}\right) \mathrm{V}_{n}\left(\Delta_{t}\right)=\mathrm{V}\left(\mathbf{v}_{0}, \ldots, \mathbf{v}_{n}\right) \cdot \int_{C_{t}} e^{-\epsilon \Phi / 2} d x_{0} \ldots d x_{n} .
$$

Since

$$
\begin{aligned}
C_{t} & =\left\{\lambda_{0} \mathbf{v}_{0}(t)+\cdots+\lambda_{n} \mathbf{v}_{n}(t) \mid \lambda_{0} \geq 0, \ldots, \lambda_{n} \geq 0\right\} \\
& =\left\{x_{0} \mathbf{v}_{0}+\cdots+x_{n} \mathbf{v}_{n} \mid x_{0}+x_{1} t \geq 0, x_{1} \geq 0, \ldots, x_{n} \geq 0\right\}
\end{aligned}
$$


we have

$$
\begin{aligned}
2^{\frac{n-1}{2}} \Gamma\left(\frac{n+1}{2}\right) \mathrm{V}_{n}\left(\Delta_{t}\right)=\mathrm{V}\left(\mathbf{v}_{0}, \ldots, \mathbf{v}_{n}\right) \\
\\
\quad \cdot \int_{x_{1}=0}^{+\infty} \int_{x_{0}=-x_{1}}^{+\infty} \int_{x_{2}=0}^{+\infty} \cdots \int_{x_{n}=0}^{+\infty} e^{-\epsilon \Phi / 2} d x_{0} \ldots d x_{n}
\end{aligned}
$$

and differentiating we obtain

$$
\begin{aligned}
\left.2^{\frac{n-1}{2}} \Gamma\left(\frac{n+1}{2}\right) \frac{d \mathrm{~V}_{n}\left(\Delta_{t}\right)}{d t}\right|_{t=0}=\mathrm{V}\left(\mathbf{v}_{0}, \ldots, \mathbf{v}_{n}\right) \\
\\
\left.\cdot \int_{x_{1}=0}^{+\infty} \int_{x_{2}=0}^{+\infty} \cdots \int_{x_{n}=0}^{+\infty} x_{1} e^{-\epsilon \Phi / 2} d x_{1} \ldots d x_{n}\right|^{x_{0}=0} .
\end{aligned}
$$

Let us now consider the dual basis $\left\{\mathbf{w}_{0}, \ldots, \mathbf{w}_{n}\right\}$ of $\left\{\mathbf{v}_{0}, \ldots, \mathbf{v}_{n}\right\}$, which satisfies the relation $\left\langle\mathbf{v}_{i}, \mathbf{w}_{j}\right\rangle=\delta_{i j}$. Taking multiples of the vectors $\mathbf{v}_{i}$ if necessary, we can always assume that $\left|\mathbf{w}_{j}\right|=1$ for $j=0, \ldots, n$. Denote by $\left(x_{0}, \ldots, x_{n}\right)$ the coordinates in the basis $\left\{\mathbf{v}_{0}, \ldots, \mathbf{v}_{n}\right\}$, and by $\left(y_{0}, \ldots, y_{n}\right)$ the coordinates in the dual basis $\left\{\mathbf{w}_{0}, \ldots, \mathbf{w}_{n}\right\}$. Then the following relation holds:

$$
x_{i}=\sum_{j=0}^{n}\left\langle\mathbf{w}_{i}, \mathbf{w}_{j}\right\rangle y_{j} \quad y_{i}=\sum_{j=0}^{n}\left\langle\mathbf{v}_{i}, \mathbf{v}_{j}\right\rangle x_{j}
$$

Now the quadratic form of $\mathbb{R}_{q}^{n+1}$ is $\Phi=\sum_{i, j=0}^{n}\left\langle\mathbf{v}_{i}, \mathbf{v}_{j}\right\rangle x_{i} x_{j}$. Therefore,

$$
\frac{\partial \Phi}{\partial x_{i}}=2 \sum_{j=0}^{n}\left\langle\mathbf{v}_{i}, \mathbf{v}_{j}\right\rangle x_{j}=2 y_{i}
$$

From (4) we deduce that, inside the hyperplane $x_{0}=0$,

$$
\left\{\begin{array}{l}
x_{1}=\sum_{j=0}^{n}\left\langle\mathbf{w}_{1}, \mathbf{w}_{j}\right\rangle y_{j} \\
x_{0}=\left\langle\mathbf{w}_{0}, \mathbf{w}_{0}\right\rangle y_{0}+\sum_{i=1}^{n}\left\langle\mathbf{w}_{0}, \mathbf{w}_{i}\right\rangle y_{i}=0 .
\end{array}\right.
$$

Hence

$$
\begin{aligned}
x_{1} & =\sum_{i=1}^{n}\left(\left\langle\mathbf{w}_{1}, \mathbf{w}_{i}\right\rangle-\frac{\left\langle\mathbf{w}_{0}, \mathbf{w}_{1}\right\rangle\left\langle\mathbf{w}_{0}, \mathbf{w}_{i}\right\rangle}{\left\langle\mathbf{w}_{0}, \mathbf{w}_{0}\right\rangle}\right) y_{i} \\
& =\sum_{i=1}^{n}\left(\left\langle\mathbf{w}_{1}, \mathbf{w}_{i}\right\rangle-\frac{\left\langle\mathbf{w}_{0}, \mathbf{w}_{1}\right\rangle\left\langle\mathbf{w}_{0}, \mathbf{w}_{i}\right\rangle}{\left\langle\mathbf{w}_{0}, \mathbf{w}_{0}\right\rangle}\right) \frac{\partial(\Phi / 2)}{\partial x_{i}} .
\end{aligned}
$$


For every $t \in \mathbb{R}$ let $\left\{\mathbf{w}_{0}(t), \ldots, \mathbf{w}_{n}(t)\right\}$ be the dual basis of $\left\{\mathbf{v}_{0}(t), \ldots\right.$, $\left.\mathbf{v}_{n}(t)\right\}$. It is easy to check that $\left\{\begin{array}{l}\mathbf{w}_{0}(t)=\mathbf{w}_{0}+t \mathbf{w}_{1} \\ \mathbf{w}_{i}(t)=\mathbf{w}_{i} \text { for } 1 \leq i \leq n\end{array}\right.$. Now define for every $i \in\{1, \ldots, n\}$ the function

$$
f_{0 i}(t)=\frac{\left\langle\mathbf{w}_{0}(t), \mathbf{w}_{i}(t)\right\rangle}{\left|\mathbf{w}_{0}(t)\right| \cdot\left|\mathbf{w}_{i}(t)\right|}
$$

Considering that $\left|\mathbf{w}_{j}\right|=1$ for all $j$, we obtain on differentiating that

$$
\left.\frac{d f_{0 i}(t)}{d t}\right|_{t=0}=\left\langle\mathbf{w}_{1}, \mathbf{w}_{i}\right\rangle-\frac{\left\langle\mathbf{w}_{0}, \mathbf{w}_{1}\right\rangle\left\langle\mathbf{w}_{0}, \mathbf{w}_{i}\right\rangle}{\left\langle\mathbf{w}_{0}, \mathbf{w}_{0}\right\rangle} .
$$

Therefore,

$$
x_{1}=\left.\sum_{i=1}^{n} \frac{d f_{0 i}(t)}{d t}\right|_{t=0} \cdot \frac{\partial(\Phi / 2)}{\partial x_{i}}
$$

and plugging this into (3) it follows that

$$
\begin{gathered}
\left.2^{\frac{n-1}{2}} \Gamma\left(\frac{n+1}{2}\right) \frac{d \mathrm{~V}_{n}\left(\Delta_{t}\right)}{d t}\right|_{t=0} \\
=\left.\mathrm{V}\left(\mathbf{v}_{0}, \ldots, \mathbf{v}_{n}\right) \cdot \int_{0}^{+\infty} \ldots \int_{0}^{+\infty} \sum_{i=1}^{n} \frac{d f_{0 i}(t)}{d t}\right|_{t=0} \\
\left.\cdot \frac{\partial(\Phi / 2)}{\partial x_{i}} e^{-\epsilon \Phi / 2} d x_{1} \ldots d x_{n}\right|^{x_{0}=0} \\
=-\left.\epsilon \cdot \mathrm{V}\left(\mathbf{v}_{0}, \ldots, \mathbf{v}_{n}\right) \cdot \sum_{i=1}^{n} \frac{d f_{0 i}(t)}{d t}\right|_{t=0} \\
\left.\cdot \int_{0}^{+\infty} \ldots \int_{0}^{+\infty} \frac{\partial\left(e^{-\epsilon \Phi / 2}\right)}{\partial x_{i}} d x_{1} \ldots d x_{n}\right|^{x_{0}=0} \\
=\left.\epsilon \cdot \mathrm{V}\left(\mathbf{v}_{0}, \ldots, \mathbf{v}_{n}\right) \cdot \sum_{i=1}^{n} \frac{d f_{0 i}(t)}{d t}\right|_{t=0} \\
\left.\cdot \int_{0}^{+\infty} \ldots \int_{0}^{+\infty} e^{-\epsilon \Phi / 2} d x_{1} \ldots d x_{n}\right|^{x_{0}=x_{i}=0} .
\end{gathered}
$$

Now let us observe that the volume of the codimension 2 face of $\Delta, F_{0 i}=$ $\left\{x_{1} \mathbf{v}_{1}+\cdots+x_{n} \mathbf{v}_{n} \mid x_{1} \geq 0, \ldots, x_{n} \geq 0 ; x_{i}=0\right\} \cap S_{q}^{n}(\epsilon)$, can also be computed using the relation (2) in the $(n-1)$-dimensional subspace of $\mathbb{R}_{q}^{n+1}$ 
spanned by $\mathbf{v}_{1}, \ldots, \widehat{\mathbf{v}}_{i}, \ldots, \mathbf{v}_{n}$. In other words,

$$
\begin{aligned}
2^{\frac{n-3}{2}} \Gamma\left(\frac{n-1}{2}\right) & \mathrm{V}_{n-2}\left(F_{0 i}\right)=\mathrm{V}\left(\mathbf{v}_{1}, \ldots, \widehat{\mathbf{v}}_{i}, \ldots, \mathbf{v}_{n}\right) \\
& \left.\cdot \int_{0}^{+\infty} \cdots \int_{0}^{+\infty} e^{-\epsilon \Phi / 2} d x_{1} \ldots d \widehat{x}_{i} \ldots d x_{n}\right|^{x_{0}=x_{i}=0} .
\end{aligned}
$$

Substituting this last expression in (5), we get

$$
\begin{aligned}
& \left.2^{\frac{n-1}{2}} \Gamma\left(\frac{n+1}{2}\right) \frac{d \mathrm{~V}_{n}\left(\Delta_{t}\right)}{d t}\right|_{t=0} \\
& =\left.\epsilon \sum_{i=1}^{n} \frac{\mathrm{V}\left(\mathbf{v}_{0}, \ldots, \mathbf{v}_{n}\right)}{\mathrm{V}\left(\mathbf{v}_{1}, \ldots, \widehat{\mathbf{v}}_{i}, \ldots, \mathbf{v}_{n}\right)} \cdot \frac{d f_{0 i}(t)}{d t}\right|_{t=0} 2^{\frac{n-3}{2}} \cdot \Gamma\left(\frac{n-1}{2}\right) \mathrm{V}_{n-2}\left(F_{0 i}\right)
\end{aligned}
$$

and since the gamma function has the property that $\Gamma\left(\frac{n+1}{2}\right)=$ $\frac{n-1}{2} \Gamma\left(\frac{n-1}{2}\right)$, it follows that

$$
\begin{aligned}
& \left.\frac{d \mathrm{~V}_{n}\left(\Delta_{t}\right)}{d t}\right|_{t=0} \\
& =\left.\frac{\epsilon}{n-1} \sum_{i=1}^{n} \frac{\mathrm{V}\left(\mathbf{v}_{0}, \ldots, \mathbf{v}_{n}\right)}{\mathrm{V}\left(\mathbf{v}_{1}, \ldots, \widehat{\mathbf{v}}_{i}, \ldots, \mathbf{v}_{n}\right)} \cdot \frac{d f_{0 i}(t)}{d t}\right|_{t=0} \cdot \mathrm{V}_{n-2}\left(F_{0 i}\right) .
\end{aligned}
$$

Let us now make some elementary observations about the volume of a parallelepiped in $\mathbb{R}_{q}^{n+1}$, which will allow us to simplify the factor $\frac{\mathrm{V}\left(\mathbf{v}_{0}, \ldots, \mathbf{v}_{n}\right)}{\mathrm{V}\left(\mathbf{v}_{1}, \ldots, \widehat{\mathbf{v}}_{i}, \ldots, \mathbf{v}_{n}\right)}$ in the last expression.

(i) The volume $\mathrm{V}\left(\mathbf{v}_{0}, \ldots, \mathbf{v}_{n}\right)$ is the same as the euclidean volume of the parallelepiped determined in $\mathbb{R}^{n+1}$ by the vectors $\mathbf{v}_{0}, \ldots, \mathbf{v}_{n}$ (because the matrix $J_{q}$ of the quadratic form of $\mathbb{R}_{q}^{n+1}$ has determinant \pm 1 ). Hence $\mathrm{V}\left(\mathbf{v}_{0}, \ldots, \mathbf{v}_{n}\right)$ is the absolute value of the determinant of the matrix having as columns the coordinates of the vectors $\mathbf{v}_{0}, \ldots, \mathbf{v}_{n}$ in the standard basis of $\mathbb{R}_{q}^{n+1}$. It also equals $\sqrt{\left|\operatorname{det}\left(\left\langle\mathbf{v}_{i}, \mathbf{v}_{j}\right\rangle\right)_{i, j}\right|}$.

(ii) Let $\left\{\mathbf{w}_{0}, \ldots, \mathbf{w}_{n}\right\}$ be the dual basis of $\left\{\mathbf{v}_{0}, \ldots, \mathbf{v}_{n}\right\}$, and let $M$ (resp. $N)$ be the matrix having as columns the coordinates of $\mathbf{v}_{0}, \ldots, \mathbf{v}_{n}$ (resp. $\mathbf{w}_{0}, \ldots, \mathbf{w}_{n}$ ). Then $M^{t} \cdot J_{q} \cdot N$ is the identity matrix, so $|\operatorname{det}(N)|=|\operatorname{det}(M)|^{-1}$ and

$$
\mathrm{V}\left(\mathbf{w}_{0}, \ldots, \mathbf{w}_{n}\right)=\frac{1}{\mathrm{~V}\left(\mathbf{v}_{0}, \ldots, \mathbf{v}_{n}\right)}
$$

(iii) For any given $k$ between 0 and $n-1$, let $\mathbf{v}_{0}^{\prime}, \ldots, \mathbf{v}_{k}^{\prime}$ be the orthogonal projections of the vectors $\mathbf{v}_{0}, \ldots, \mathbf{v}_{k}$ onto the subspace spanned 
by $\mathbf{w}_{0}, \ldots, \mathbf{w}_{k}$ (which is itself orthogonal to the remaining vectors $\left.\mathbf{v}_{k+1}, \ldots, \mathbf{v}_{n}\right)$. Then for every $i \in\{0, \ldots, k\}, \mathbf{v}_{i}^{\prime}-\mathbf{v}_{i}$ is a linear combination of the vectors $\mathbf{v}_{k+1}, \ldots, \mathbf{v}_{n}$, and therefore

$$
\begin{aligned}
\mathrm{V}\left(\mathbf{v}_{0}, \ldots, \mathbf{v}_{n}\right) & =\mathrm{V}\left(\mathbf{v}_{0}^{\prime}, \ldots, \mathbf{v}_{k}^{\prime}, \mathbf{v}_{k+1}, \ldots, \mathbf{v}_{n}\right) \\
& =\mathrm{V}\left(\mathbf{v}_{0}^{\prime}, \ldots, \mathbf{v}_{k}^{\prime}\right) \cdot \mathrm{V}\left(\mathbf{v}_{k+1}, \ldots, \mathbf{v}_{n}\right)
\end{aligned}
$$

(the last equality being a consequence of the orthogonality). On the other hand, it is inmediately checked that $\left\{\mathbf{w}_{0}, \ldots, \mathbf{w}_{k}\right\}$ is the dual basis of $\left\{\mathbf{v}_{0}^{\prime}, \ldots, \mathbf{v}_{k}^{\prime}\right\}$ inside the subspace spanned by $\mathbf{w}_{0}, \ldots, \mathbf{w}_{k}$. Hence from (ii) follows that

$$
\mathrm{V}\left(\mathbf{v}_{0}, \ldots, \mathbf{v}_{n}\right)=\frac{\mathrm{V}\left(\mathbf{v}_{k+1}, \ldots, \mathbf{v}_{n}\right)}{\mathrm{V}\left(\mathbf{w}_{0}, \ldots, \mathbf{w}_{k}\right)} .
$$

Using this last property in the particular case of expression (6) we get

$$
\begin{aligned}
& \left.\frac{d \mathrm{~V}_{n}\left(\Delta_{t}\right)}{d t}\right|_{t=0} \\
& =\left.\frac{\epsilon}{n-1} \sum_{i=1}^{n} \frac{1}{\mathrm{~V}\left(\mathbf{w}_{0}, \mathbf{w}_{i}\right)} \cdot \frac{d f_{0 i}(t)}{d t}\right|_{t=0} \cdot \mathrm{V}_{n-2}\left(F_{0 i}\right) \\
& =\frac{\epsilon}{n-1} \sum_{i=1}^{n} \frac{1}{\sqrt{\left|\left\langle\mathbf{w}_{0}, \mathbf{w}_{0}\right\rangle \cdot\left\langle\mathbf{w}_{i}, \mathbf{w}_{i}\right\rangle-\left\langle\mathbf{w}_{0}, \mathbf{w}_{i}\right\rangle^{2}\right|}} \\
& \left.\quad \cdot \frac{d f_{0 i}(t)}{d t}\right|_{t=0} \cdot \mathrm{V}_{n-2}\left(F_{0 i}\right) .
\end{aligned}
$$

Before we can proceed further to arrive at a Schläfli formula, we need a definition of dihedral angle in semi-riemannian geometry. A natural definition has been given by J-M. Schlenker (cf. $\left[\mathbf{S c h l e}_{1}\right]$, [Schle 2$]$ ), in which the dihedral angle can take complex values. This definition, together with a suitable definition of semi-riemannian volume (also complex-valued), provides a Schläfli formula and Gauss-Bonnet formulas in the semi-riemannian case which are identical to the corresponding spherical formulas (cf. [Schle 1 ]).

However, here we are interested in defining the dihedral angle $\alpha_{0 i}(t)$ at the face $F_{0 i}(t)$ of $\Delta_{t}$ as a real number, in such a way that

$$
\left.\frac{d \alpha_{0 i}(t)}{d t}\right|_{t=0}=\left.\frac{1}{\sqrt{\left|\left\langle\mathbf{w}_{0}, \mathbf{w}_{0}\right\rangle \cdot\left\langle\mathbf{w}_{i}, \mathbf{w}_{i}\right\rangle-\left\langle\mathbf{w}_{0}, \mathbf{w}_{i}\right\rangle^{2}\right|}} \cdot \frac{d f_{0 i}(t)}{d t}\right|_{t=0}
$$

where $f_{0 i}(t)=\frac{\left\langle\mathbf{w}_{0}(t), \mathbf{w}_{i}(t)\right\rangle}{\left|\mathbf{w}_{0}(t)\right| \cdot\left|\mathbf{w}_{i}(t)\right|}$.

\subsection{A definition of dihedral angle in semi-riemannian geometry.}

Consider the 2 -dimensional vector subspace of $\mathbb{R}_{q}^{n+1}$ spanned by the vectors $\mathbf{w}_{0}$ and $\mathbf{w}_{i}$, which is orthogonal to the codimension 2 subspace $\left\langle C_{0 i}\right\rangle$ spanned by $\mathbf{v}_{1}, \ldots, \widehat{\mathbf{v}}_{i}, \ldots, \mathbf{v}_{n}$. The restriction of the quadratic form of $\mathbb{R}_{q}^{n+1}$ to 
this plane is non-degenerate, because of the condition $(*)$ imposed upon the simplex $\Delta$ (cf. Remark 1.2). If it is a (positive or negative) definite quadratic form, then the dihedral angle at the face $F_{0 i}$ can be defined in the usual manner, as

$$
\alpha_{0 i}=\pi-\arccos \left(\frac{\left\langle\mathbf{w}_{0}, \mathbf{w}_{i}\right\rangle}{\left|\mathbf{w}_{0}\right| \cdot\left|\mathbf{w}_{i}\right|}\right)=\pi-\arccos \left(f_{0 i}\right) .
$$

However, this orthogonal plane can also have a Lorentz metric, so we need to define the angle between two non-null vectors in the Lorentz-Minkowski plane $\mathbb{R}_{1}^{2}$. The difficulty lies in the fact that the set of unit vectors in $\mathbb{R}_{1}^{2}$ is not bounded. In the Euclidean plane $\mathbb{R}^{2}$, the angle between two unit vectors $\mathbf{w}_{1}, \mathbf{w}_{2}$ can be defined as the length of the arc they subtend on the unit sphere $\mathbb{S}^{1}$. If we try to define the angle between two unit vectors in $\mathbb{R}_{1}^{2}$ in an analogous manner, it can happen that the arc they subtend on the set of all unit vectors has infinite branches.

A natural way to solve the problem is the following. Since the hyperbola $\mathcal{H}^{-}$corresponding to timelike unit vectors in $\mathbb{R}_{1}^{2}$ has a positive definite metric, while the hyperbola $\mathcal{H}^{+}$corresponding to spacelike unit vectors has a negative definite metric, we will assign positive length to all arcs contained in $\mathcal{H}^{-}$and negative length to all arcs contained in $\mathcal{H}^{+}$. Given two non-null unit vectors $\mathbf{w}_{1}, \mathbf{w}_{2}$ in $\mathbb{R}_{1}^{2}$, consider the arc (possibly with infinite branches) subtended by them in $\mathcal{H}^{-} \cup \mathcal{H}^{+}$, and let $l_{r}$ be the sum of the lengths (with the above sign convention) of the portions of this arc contained inside the Euclidean ball of radius $r$ in $\mathbb{R}^{2}$. Then define the angle between $\mathbf{w}_{1}$ and $\mathbf{w}_{2}$ as the limit of $l_{r}$ as $r$ tends to infinity. It is easy to check that this is equivalent to the following definition.

Definition 1.2. Given two non-null vectors $\mathbf{w}_{1}, \mathbf{w}_{2}$ in $\mathbb{R}_{1}^{2}$, the angle between $\mathbf{w}_{1}$ and $\mathbf{w}_{2}$ is defined by

$$
\begin{cases}\operatorname{ang}\left(\mathbf{w}_{1}, \mathbf{w}_{2}\right)=\operatorname{arccosh}\left(\frac{-\left\langle\mathbf{w}_{1}, \mathbf{w}_{2}\right\rangle}{\left|\mathbf{w}_{1}\right| \cdot\left|\mathbf{w}_{2}\right|}\right) & \text { if }\left\langle\mathbf{w}_{1}, \mathbf{w}_{1}\right\rangle \cdot\left\langle\mathbf{w}_{2}, \mathbf{w}_{2}\right\rangle>0 \\ & \text { and }\left\langle\mathbf{w}_{1}, \mathbf{w}_{2}\right\rangle<0 ; \\ \operatorname{ang}\left(\mathbf{w}_{1}, \mathbf{w}_{2}\right)=-\operatorname{arccosh}\left(\frac{\left\langle\mathbf{w}_{1}, \mathbf{w}_{2}\right\rangle}{\left|\mathbf{w}_{1}\right| \cdot\left|\mathbf{w}_{2}\right|}\right) & \text { if }\left\langle\mathbf{w}_{1}, \mathbf{w}_{1}\right\rangle \cdot\left\langle\mathbf{w}_{2}, \mathbf{w}_{2}\right\rangle>0 \\ & \text { and }\left\langle\mathbf{w}_{1}, \mathbf{w}_{2}\right\rangle>0 ; \\ \operatorname{ang}\left(\mathbf{w}_{1}, \mathbf{w}_{2}\right)=-\operatorname{arcsinh}\left(\frac{\left\langle\mathbf{w}_{1}, \mathbf{w}_{2}\right\rangle}{\left|\mathbf{w}_{1}\right| \cdot\left|\mathbf{w}_{2}\right|}\right) & \text { if }\left\langle\mathbf{w}_{1}, \mathbf{w}_{1}\right\rangle \cdot\left\langle\mathbf{w}_{2}, \mathbf{w}_{2}\right\rangle<0 .\end{cases}
$$

This definition is consistent with the standard one for the hyperbolic angle between two timelike vectors lying in the same timecone of a Lorentz vector space (cf. $\left[\mathbf{O}^{\prime} \mathbf{N}\right]$ ), and it is also the adequate one for our purpose. 
Since with our sign convention we have assigned zero length to the set of all unit vectors in $\mathbb{R}_{1}^{2}$, the following definition of dihedral angle is also natural.

Definition 1.3. Given two non-null vectors $\mathbf{w}_{1}, \mathbf{w}_{2}$ in $\mathbb{R}_{q}^{n+1}$ which span a Lorentz plane, the dihedral angle $\alpha$ at the edge of the dihedron $\left\{\mathbf{v} \in \mathbb{R}_{q}^{n+1}\right.$ : $\left.\left\langle\mathbf{v}, \mathbf{w}_{1}\right\rangle \geq 0,\left\langle\mathbf{v}, \mathbf{w}_{2}\right\rangle \geq 0\right\}$ is defined as $\alpha=-\operatorname{ang}\left(\mathbf{w}_{1}, \mathbf{w}_{2}\right)$.

\subsection{A Schläfli differential formula.}

Theorem 1.1. Let $S_{q}^{n}(\epsilon)$ be a connected component of the central unit hyperquadric of sign $\epsilon$ of $\mathbb{R}_{q}^{n+1}$, and let $\Delta$ be a family of $n$-simplices in $S_{q}^{n}(\epsilon)$ varying differentiably with respect to one or more parameters, and such that all their faces of codimension 1 and 2 have a non-degenerate induced metric. Then the differential of their volume $\mathrm{V}_{n}(\Delta)$ satisfies the following equality:

$$
d \mathrm{~V}_{n}(\Delta)=\frac{\epsilon}{n-1} \sum_{F} \mathrm{~V}_{n-2}(F) d \alpha_{F}
$$

where the sum is taken over all codimension 2 faces $F$ of $\Delta, V_{n-2}(F)$ is the $((n-2)$-dimensional $)$ volume of the face $F$ and $\alpha_{F}$ is the dihedral angle at the face $F$. (When $n-2=0$, we set $\mathrm{V}_{0}(F)=1$.)

Proof. Let us continue the proof at the point where we had arrived in subsection 1.3. Our starting point was an $n$-simplex

$$
\Delta=\left\{x_{0} \mathbf{v}_{0}+\cdots+x_{n} \mathbf{v}_{n} \mid x_{0} \geq 0, \ldots, x_{n} \geq 0\right\} \cap S_{q}^{n}(\epsilon)
$$

(where the basis $\left\{\mathbf{v}_{0}, \ldots, \mathbf{v}_{n}\right\}$ is chosen so that its dual basis is formed by unit vectors $\mathbf{w}_{i}$ ), and we deformed it by moving one of its vertices, $\mathbf{v}_{1}$, towards another one, $\mathbf{v}_{0}$, keeping all the others fixed. More precisely, for every $t$ sufficiently close to 0 we defined the $n$-simplex

$\Delta_{t}=\left\{\lambda_{0} \mathbf{v}_{0}+\lambda_{1}\left(\mathbf{v}_{1}-t \mathbf{v}_{0}\right)+\lambda_{2} \mathbf{v}_{2}+\cdots+\lambda_{n} \mathbf{v}_{n} \mid \lambda_{0} \geq 0, \ldots, \lambda_{n} \geq 0\right\} \cap S_{q}^{n}(\epsilon)$.

The only dihedral angles that change are the angles at the edges of the face opposite to the vertex $\mathbf{v}_{0}$, that is, the dihedral angles $\alpha_{0 i}(t)$ at the codimension 2 faces of $\Delta_{t}$ of the form $F_{0 i}(t)(i=1, \ldots, n)$. Therefore, what we want to prove is that

$$
\left.\frac{d \mathrm{~V}_{n}\left(\Delta_{t}\right)}{d t}\right|_{t=0}=\left.\frac{\epsilon}{n-1} \sum_{i=1}^{n} \mathrm{~V}_{n-2}\left(F_{0 i}\right) \cdot \frac{d \alpha_{0 i}(t)}{d t}\right|_{t=0} .
$$

In subsection 1.3 we showed that

$$
\begin{aligned}
& \left.\frac{d \mathrm{~V}_{n}\left(\Delta_{t}\right)}{d t}\right|_{t=0} \\
& =\left.\frac{\epsilon}{n-1} \sum_{i=1}^{n} \mathrm{~V}_{n-2}\left(F_{0 i}\right) \cdot \frac{1}{\sqrt{\left|\left\langle\mathbf{w}_{0}, \mathbf{w}_{0}\right\rangle \cdot\left\langle\mathbf{w}_{i}, \mathbf{w}_{i}\right\rangle-\left\langle\mathbf{w}_{0}, \mathbf{w}_{i}\right\rangle^{2}\right|}} \cdot \frac{d f_{0 i}(t)}{d t}\right|_{t=0}
\end{aligned}
$$


where $f_{0 i}(t)=\frac{\left\langle\mathbf{w}_{0}(t), \mathbf{w}_{i}(t)\right\rangle}{\left|\mathbf{w}_{0}(t)\right| \cdot\left|\mathbf{w}_{i}(t)\right|}$. Hence it only remains to prove that

$$
\left.\frac{d \alpha_{0 i}(t)}{d t}\right|_{t=0}=\left.\frac{1}{\sqrt{\left|\left\langle\mathbf{w}_{0}, \mathbf{w}_{0}\right\rangle \cdot\left\langle\mathbf{w}_{i}, \mathbf{w}_{i}\right\rangle-\left\langle\mathbf{w}_{0}, \mathbf{w}_{i}\right\rangle^{2}\right|}} \cdot \frac{d f_{0 i}(t)}{d t}\right|_{t=0} .
$$

We need to distinguish several cases:

- Case 1. If $\left\langle\mathbf{w}_{0}, \mathbf{w}_{0}\right\rangle \cdot\left\langle\mathbf{w}_{i}, \mathbf{w}_{i}\right\rangle-\left\langle\mathbf{w}_{0}, \mathbf{w}_{i}\right\rangle^{2}>0$, then for $t$ close to 0 , the plane spanned by $\mathbf{w}_{0}(t)$ and $\mathbf{w}_{i}(t)$ has a definite metric, and the dihedral angle at the face $F_{0 i}(t)$ is $\alpha_{0 i}(t)=\pi-\arccos \left(f_{0 i}(t)\right)$. Hence $\left.\frac{d \alpha_{0 i}(t)}{d t}\right|_{t=0}=\left.\frac{1}{\sqrt{1-f_{0 i}^{2}(0)}} \cdot \frac{d f_{0 i}(t)}{d t}\right|_{t=0}$

- Case 2. If $\left\langle\mathbf{w}_{0}, \mathbf{w}_{0}\right\rangle \cdot\left\langle\mathbf{w}_{i}, \mathbf{w}_{i}\right\rangle-\left\langle\mathbf{w}_{0}, \mathbf{w}_{i}\right\rangle^{2}<0$, then for $t$ close to 0 , the plane spanned by $\mathbf{w}_{0}(t)$ and $\mathbf{w}_{i}(t)$ has a Lorentz metric. There are three possibilities:

(i) $\left\langle\mathbf{w}_{0}, \mathbf{w}_{0}\right\rangle \cdot\left\langle\mathbf{w}_{i}, \mathbf{w}_{i}\right\rangle>0$ and $\left\langle\mathbf{w}_{0}, \mathbf{w}_{i}\right\rangle<0$.

Then $\alpha_{0 i}(t)=-\operatorname{arccosh}\left(-f_{0 i}(t)\right)$ and

$$
\left.\frac{d \alpha_{0 i}(t)}{d t}\right|_{t=0}=\left.\frac{1}{\sqrt{f_{0 i}^{2}(0)-1}} \cdot \frac{d f_{0 i}(t)}{d t}\right|_{t=0} .
$$

(ii) $\left\langle\mathbf{w}_{0}, \mathbf{w}_{0}\right\rangle \cdot\left\langle\mathbf{w}_{i}, \mathbf{w}_{i}\right\rangle>0$ and $\left\langle\mathbf{w}_{0}, \mathbf{w}_{i}\right\rangle>0$.

Now $\alpha_{0 i}(t)=\operatorname{arccosh}\left(f_{0 i}(t)\right)$ and

$$
\left.\frac{d \alpha_{0 i}(t)}{d t}\right|_{t=0}=\left.\frac{1}{\sqrt{f_{0 i}^{2}(0)-1}} \cdot \frac{d f_{0 i}(t)}{d t}\right|_{t=0} .
$$

(iii) $\left\langle\mathbf{w}_{0}, \mathbf{w}_{0}\right\rangle \cdot\left\langle\mathbf{w}_{i}, \mathbf{w}_{i}\right\rangle<0$.

Now $\alpha_{0 i}(t)=\operatorname{arcsinh}\left(f_{0 i}(t)\right)$ and

$$
\left.\frac{d \alpha_{0 i}(t)}{d t}\right|_{t=0}=\left.\frac{1}{\sqrt{1+f_{0 i}^{2}(0)}} \cdot \frac{d f_{0 i}(t)}{d t}\right|_{t=0} .
$$

Taking into account the fact that all the vectors $\mathbf{w}_{i}$ have norm 1 , we see that in all these cases

$$
\left.\frac{d \alpha_{0 i}(t)}{d t}\right|_{t=0}=\left.\frac{1}{\sqrt{\left|\left\langle\mathbf{w}_{0}, \mathbf{w}_{0}\right\rangle \cdot\left\langle\mathbf{w}_{i}, \mathbf{w}_{i}\right\rangle-\left\langle\mathbf{w}_{0}, \mathbf{w}_{i}\right\rangle^{2}\right|}} \cdot \frac{d f_{0 i}(t)}{d t}\right|_{t=0} .
$$

Therefore,

$$
\left.\frac{d \mathrm{~V}_{n}\left(\Delta_{t}\right)}{d t}\right|_{t=0}=\left.\frac{\epsilon}{n-1} \sum_{i=1}^{n} \mathrm{~V}_{n-2}\left(F_{0 i}\right) \cdot \frac{d \alpha_{0 i}(t)}{d t}\right|_{t=0} .
$$




\section{Dual volume of a tetrahedron in 3-dimensional hyperbolic space. (A formula of Santaló, revisited.)}

We define the dual volume of a hyperbolic $n$-simplex $\Delta$ in $\mathbb{H}^{n}$ as the "measure" of the set of hyperplanes of $\mathbb{H}^{n}$ intersecting it. There are two natural ways to define a measure on the set $\mathcal{P}^{n}$ of hyperplanes of $\mathbb{H}^{n}$. On the one hand, $\mathcal{P}^{n}$ can be identified with the Lie group $O(1, n) / \mathcal{J}$, where $\mathcal{J}$ is the subgroup of $O(1, n)$ formed by all transformations leaving a given hyperplane of $\mathbb{H}^{n}$ invariant. This Lie group has a left-invariant volume form, which defines a measure on $\mathcal{P}^{n}$ (cf. $\left.\left[\mathbf{S a}_{2}\right]\right)$. On the other hand, the de Sitter sphere $\mathbb{S}_{1}^{n}(1)$ (which we will simply denote by $\mathbb{S}_{1}^{n}$ from now on) is a double cover of $\mathcal{P}^{n}$, via the map associating to each point $\mathbf{v} \in \mathbb{S}_{1}^{n}$ the hyperplane of $\mathbb{H}^{n}$ orthogonal to $\mathbf{v}$. Since the volume form of $\mathbb{S}_{1}^{n}$ is invariant under the antipodal map, it also induces a measure on $\mathcal{P}^{n}$. It can be checked that both definitions coincide. Hence the dual volume of a hyperbolic $n$-simplex can be computed as the volume of a certain subset of the de Sitter sphere.

\subsection{Polar dual and complementary dual of a hyperbolic simplex.}

Let $\Delta$ be an $n$-simplex in hyperbolic space $\mathbb{H}^{n}$. Let $\left\{\mathbf{v}_{0}, \ldots, \mathbf{v}_{n}\right\}$ be a basis of $\mathbb{R}_{1}^{n+1}$ such that $\Delta=\left\{x_{0} \mathbf{v}_{0}+\cdots+x_{n} \mathbf{v}_{n} \mid x_{0} \geq 0, \ldots, x_{n} \geq 0\right\} \cap \mathbb{H}^{n}$, and let $\left\{\mathbf{w}_{0}, \ldots, \mathbf{w}_{n}\right\}$ be the dual basis, which is formed by spacelike vectors. We will associate to $\Delta$ the following two subsets of the de Sitter sphere $\mathbb{S}_{1}^{n}$ :

(i) the polar dual

$$
\Delta^{*}=\left\{x_{0} \mathbf{w}_{0}+\cdots+x_{n} \mathbf{w}_{n} \mid x_{0} \geq 0, \ldots, x_{n} \geq 0\right\} \cap \mathbb{S}_{1}^{n}
$$

which is the intersection of $\mathbb{S}_{1}^{n}$ with a simplicial cone of $\mathbb{R}_{1}^{n+1}$ but is not bounded; and

(ii) the complementary dual

$\Delta^{P}=\left\{x_{0} \mathbf{w}_{0}+\cdots+x_{n} \mathbf{w}_{n} \mid x_{0} \geq 0\right.$ and $x_{i} \leq 0$ for some $\left.i \in\{1, \ldots, n\}\right\} \cap \mathbb{S}_{1}^{n}$

which is a compact subset of $\mathbb{S}_{1}^{n}$ but is not a simplex.

Remark 2.1. The following properties are easily checked. The complementary dual $\Delta^{P}$ is a compact polyhedron in $\mathbb{S}_{1}^{n}$, whose faces of codimension $\geq 1$ are riemannian (i.e., they are spherical simplices). The polar dual $\Delta^{*}$ is not bounded, but all its faces of codimension $\geq 1$ are also spherical simplices. Moreover, every face of $\Delta^{*}$ of codimension $\geq 2$ is also a face of $\Delta^{P}$. The dihedral angle of $\Delta^{*}$ at a codimension 2 face is the opposite of the dihedral angle of $\Delta^{P}$ at the same face. In fact, if $F_{i j}^{*}=\left\{x_{0} \mathbf{w}_{0}+\cdots+x_{n} \mathbf{w}_{n} \mid x_{0} \geq 0, \ldots, x_{n} \geq 0 ; x_{i}=x_{j}=0\right\} \cap \mathbb{S}_{1}^{n}$ is a codimension 2 face of $\Delta^{*}$, then the dihedral angle of $\Delta^{*}$ at the face $F_{i j}^{*}$ is $-\operatorname{ang}\left(\mathbf{v}_{i}, \mathbf{v}_{j}\right)=-\operatorname{arccosh}\left(\frac{-\left\langle\mathbf{v}_{i}, \mathbf{v}_{j}\right\rangle}{\left|\mathbf{v}_{i}\right| \cdot\left|\mathbf{v}_{j}\right|}\right)$, while the dihedral angle of $\Delta^{P}$ at the same face is $\operatorname{ang}\left(\mathbf{v}_{i}, \mathbf{v}_{j}\right)$. 
In the following figure we show an example of $\Delta^{*}$ and $\Delta^{P}$ in dimension 2 , seen in the projective Klein model.

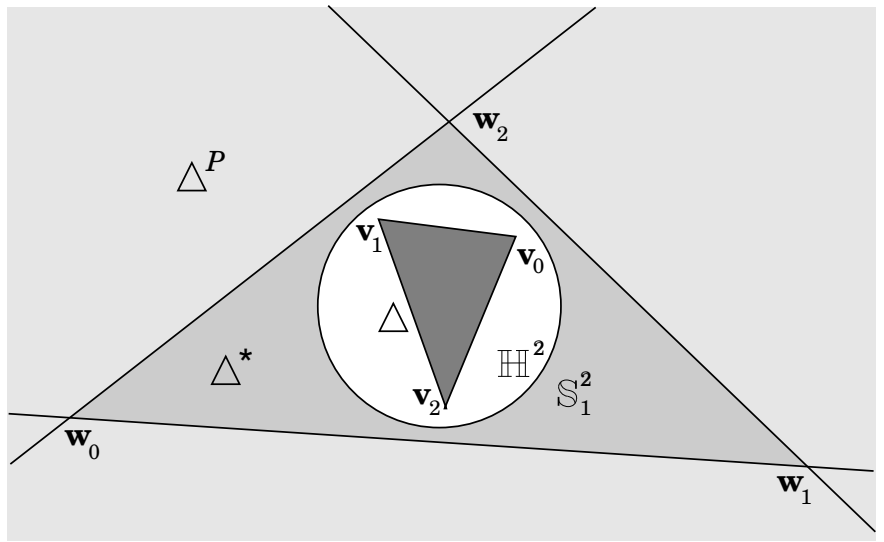

What makes the complementary dual $\Delta^{P}$ interesting is its geometric interpretation. Consider the map associating to each point $\mathbf{w}$ of $\Delta^{P}$ the hyperplane of $\mathbb{H}^{n}$ orthogonal to $\mathbf{w}$. This is a surjective map from $\Delta^{P}$ onto the set of all hyperbolic hyperplanes intersecting $\Delta$. It is not one-to-one, because there are pairs of antipodal points inside $\Delta^{P}$. However, these pairs of points are contained in the boundary of $\Delta^{P}$ and they form a subset of measure zero. Hence there is a bijection between $\Delta^{P}$ minus a subset of measure zero, and the set of all hyperbolic hyperplanes intersecting $\Delta$. Therefore the dual volume of a hyperbolic $n$-simplex is equal to the volume of its complementary dual $\Delta^{P}$. To compute this volume we use the Schläfli differential formula in the de Sitter sphere, obtained in the previous section. (Although $\Delta^{P}$ is not a simplex, we can just apply the Schläfli formula to a triangulation of $\Delta^{P}$.)

Lemma 2.1. Let $\Delta$ be a family of hyperbolic n-simplices varying differentiably with respect to one or more parameters. Then their complementary duals also vary in a differentiable manner, and the differential of their volume $\mathrm{V}_{n}\left(\Delta^{P}\right)$ satisfies the following equality:

$$
d \mathrm{~V}_{n}\left(\Delta^{P}\right)=\frac{1}{n-1} \sum_{F^{*}} \mathrm{~V}_{n-2}\left(F^{*}\right) d \alpha_{F^{*}}
$$

where the sum is taken over all codimension 2 faces $F^{*}$ of the polar dual $\Delta^{*}$ (which are also codimension 2 faces of $\left.\Delta^{P}\right), V_{n-2}\left(F^{*}\right)$ is the $((n-2)$ dimensional) volume of the face $F^{*}$ and $\alpha_{F^{*}}$ is the dihedral angle of $\Delta^{P}$ at the face $F^{*}$. (When $n-2=0$, we set $\mathrm{V}_{0}\left(F^{*}\right)=1$.)

2.2. Dual volume of a hyperbolic tetrahedron in dimension 3 . The following proposition can be found in $\left[\mathbf{S a}_{2}, \S I V .17 .5\right.$, Note 1]. Here we prove it using the Schläfli formula (compare with [Mil]). 
Proposition 2.1 (Santaló). Let $\Delta$ be a hyperbolic tetrahedron in $\mathbb{H}^{3}$, and let $\Delta^{P}$ be its complementary dual. Then the volume of $\Delta$ and its dual volume are related as follows:

$$
\mathrm{V}_{3}(\Delta)+\mathrm{V}_{3}\left(\Delta^{P}\right)=\frac{1}{2} \sum_{F}\left(\pi-\alpha_{F}\right) \mathrm{V}_{1}(F)
$$

where the sum runs over all edges $F$ of the tetrahedron $\Delta, \mathrm{V}_{1}(F)$ is the length of the edge $F$ and $\alpha_{F}$ is the dihedral angle at the edge $F$.

Proof. Let $\Delta=\left\{x_{0} \mathbf{v}_{0}+\cdots+x_{3} \mathbf{v}_{3} \mid x_{0} \geq 0, \ldots, x_{3} \geq 0\right\} \cap \mathbb{H}^{3}$, and let $\left\{\mathbf{w}_{0}, \ldots, \mathbf{w}_{3}\right\}$ be the dual basis of $\left\{\mathbf{v}_{0}, \ldots, \mathbf{v}_{3}\right\}$. The hyperbolic Schäfli formula says that

$$
d \mathrm{~V}_{3}(\Delta)=-\frac{1}{2} \sum_{0 \leq i<j \leq 3} \mathrm{~V}_{1}\left(F_{i j}\right) d \alpha_{i j}
$$

where $F_{i j}=\left\{x_{0} \mathbf{v}_{0}+\cdots+x_{3} \mathbf{v}_{3} \mid x_{0} \geq 0, \ldots, x_{3} \geq 0 ; x_{i}=x_{j}=0\right\} \cap \mathbb{H}^{3}$ and $\alpha_{i j}$ is the dihedral angle of $\Delta$ at the edge $F_{i j}$.

On the other hand, we know from Lemma 2.1 above that

$$
d \mathrm{~V}_{3}\left(\Delta^{P}\right)=\frac{1}{2} \sum_{0 \leq k<l \leq 3} \mathrm{~V}_{1}\left(F_{k l}^{*}\right) d \alpha_{k l}^{*}
$$

where $F_{k l}^{*}=\left\{x_{0} \mathbf{w}_{0}+\cdots+x_{3} \mathbf{w}_{3} \mid x_{0} \geq 0, \ldots, x_{3} \geq 0 ; x_{k}=x_{l}=0\right\} \cap \mathbb{S}_{1}^{3}$ and $\alpha_{k l}^{*}$ is the dihedral angle of $\Delta^{P}$ at the edge $F_{k l}^{*}$. By Remark 2.1, $\alpha_{k l}^{*}=$ $\operatorname{ang}\left(\mathbf{v}_{k}, \mathbf{v}_{l}\right)$.

Then the following relations hold. Given $0 \leq i<j \leq 3$, denote by $0 \leq k<l \leq 3$ the other two elements of $\{0,1,2,3\}$ different from $i, j$. Then:

$$
\mathrm{V}_{1}\left(F_{k l}^{*}\right)=\operatorname{ang}\left(\mathbf{w}_{k}, \mathbf{w}_{l}\right)=\pi-\alpha_{i j} \quad \text { and } \quad \alpha_{k l}^{*}=\operatorname{ang}\left(\mathbf{v}_{k}, \mathbf{v}_{l}\right)=\mathrm{V}_{1}\left(F_{i j}\right) .
$$

Hence

$$
\begin{aligned}
d \mathrm{~V}_{3}\left(\Delta^{P}\right)= & \frac{1}{2} \sum_{0 \leq i<j \leq 3}\left(\pi-\alpha_{i j}\right) d \mathrm{~V}_{1}\left(F_{i j}\right) \\
= & \frac{1}{2} \sum_{0 \leq i<j \leq 3}\left(\pi-\alpha_{i j}\right) d \mathrm{~V}_{1}\left(F_{i j}\right)-\frac{1}{2} \sum_{0 \leq i<j \leq 3} \mathrm{~V}_{1}\left(F_{i j}\right) d \alpha_{i j} \\
& +\frac{1}{2} \sum_{0 \leq i<j \leq 3} \mathrm{~V}_{1}\left(F_{i j}\right) d \alpha_{i j} \\
= & \frac{1}{2} d\left(\sum_{0 \leq i<j \leq 3}\left(\pi-\alpha_{i j}\right) \cdot \mathrm{V}_{1}\left(F_{i j}\right)\right)-d \mathrm{~V}_{3}(\Delta)
\end{aligned}
$$

and therefore

$$
d\left(\mathrm{~V}_{3}(\Delta)+\mathrm{V}_{3}\left(\Delta^{P}\right)\right)=d\left(\frac{1}{2} \sum_{F}\left(\pi-\alpha_{F}\right) \cdot \mathrm{V}_{1}(F)\right)
$$


where the sum extends over all edges $F$ of the tetrahedron $\Delta$. We obtain on integrating that

$$
\mathrm{V}_{3}(\Delta)+\mathrm{V}_{3}\left(\Delta^{P}\right)=\frac{1}{2} \sum_{F}\left(\pi-\alpha_{F}\right) \cdot \mathrm{V}_{1}(F)+\text { constant. }
$$

To determine the constant, consider the limiting case when $\Delta$ degenerates to a point, so that the expression $\frac{1}{2} \sum_{F}\left(\pi-\alpha_{F}\right) \mathrm{V}_{1}(F)$ tends to zero. In this case, the complementary dual $\Delta^{P}$ degenerates to a plane and $\mathrm{V}_{3}(\Delta)+$ $\mathrm{V}_{3}\left(\Delta^{P}\right)$ also tends to zero. Hence the constant is zero and the formula is proved.

In higher dimensions the relation between the volume and the dual volume of a hyperbolic $n$-simplex $\Delta$ is not so straightforward. The reason is that for $n>3$, the volume of a codimension 2 face of $\Delta$ is equal to the dihedral angle of $\Delta^{P}$ at a face of codimension $n-1 \neq 2$, which does not appear in the Schläfli formula for the volume of $\Delta^{P}$. To generalize Santaló's formula to higher dimensions, we will need some equalities that will be proved in the next section (see Proposition 3.1 below). We will also benefit from them to obtain Gauss-Bonnet formulas for $n$-simplices with riemannian faces in the de Sitter sphere $\mathbb{S}_{1}^{n}$.

\section{Gauss-Bonnet formulas in the de Sitter sphere, for simplices with riemannian faces.}

Let $\Delta$ be an $n$-simplex in the de Sitter sphere $\mathbb{S}_{1}^{n}$, such that all its faces have a riemannian induced metric. Let $\left\{\mathbf{v}_{0}, \ldots, \mathbf{v}_{n}\right\}$ be a basis of $\mathbb{R}_{1}^{n+1}$ such that $\Delta=\left\{x_{0} \mathbf{v}_{0}+\cdots+x_{n} \mathbf{v}_{n} \mid x_{0} \geq 0, \ldots, x_{n} \geq 0\right\} \cap \mathbb{S}_{1}^{n}$, and let $\left\{\mathbf{w}_{0}, \ldots, \mathbf{w}_{n}\right\}$ be the dual basis, which is formed by timelike vectors. Then we can find signs $\epsilon_{0}, \ldots, \epsilon_{n} \in\{ \pm 1\}$ such that $\epsilon_{0} \mathbf{w}_{0}, \ldots, \epsilon_{n} \mathbf{w}_{n}$ are contained in the upper timecone. We will define the polar hyperbolic simplex of $\Delta$ as

$$
\Delta^{*}=\left\{x_{0} \cdot \epsilon_{0} \mathbf{w}_{0}+\cdots+x_{n} \cdot \epsilon_{n} \mathbf{w}_{n} \mid x_{0} \geq 0, \ldots, x_{n} \geq 0\right\} \cap \mathbb{H}^{n} .
$$

Definition 3.1. Given $0 \leq r \leq n-1$ and a codimension $r+1$ face of the simplex $\Delta$,

$$
\begin{aligned}
F_{i_{0} \ldots i_{r}}=\left\{x_{0} \mathbf{v}_{0}+\cdots+x_{n} \mathbf{v}_{n} \mid x_{0} \geq 0, \ldots,\right. & x_{n} \geq 0 \\
& \left.x_{i_{0}}=\cdots=x_{i_{r}}=0\right\} \cap \mathbb{S}_{1}^{n}
\end{aligned}
$$

we define the polar angle of $\Delta$ at the face $F_{i_{0}} \ldots i_{r}$, as the $r$-dimensional hyperbolic simplex

$$
\Theta_{i_{0} \ldots i_{r}}=\left\{x_{i_{0}} \cdot \epsilon_{i_{0}} \mathbf{w}_{i_{0}}+\cdots+x_{i_{r}} \cdot \epsilon_{i_{r}} \mathbf{w}_{i_{r}} \mid x_{i_{0}} \geq 0, \ldots, x_{i_{r}} \geq 0\right\} \cap \mathbb{H}^{n}
$$


(which is a face of the polar hyperbolic simplex $\Delta^{*}$ ). We define the algebraic measure of the polar angle $\Theta_{i_{0} \ldots i_{r}}$ as:

$$
\theta_{i_{0} \ldots i_{r}}=\epsilon_{i_{0}} \cdots \epsilon_{i_{r}} \cdot \mathrm{V}_{r}\left(\Theta_{i_{0} \ldots i_{r}}\right)
$$

where $\mathrm{V}_{r}\left(\Theta_{i_{0}} \ldots i_{r}\right)$ is the ( $r$-dimensional) volume of the hyperbolic simplex $\Theta_{i_{0} \ldots i_{r}}$. When $r=0$, we set $\theta_{i}=\epsilon_{i}= \pm 1$ for $i=0, \ldots, n$.

Proposition 3.1. Let $\Delta$ be a family of n-simplices in $\mathbb{S}_{1}^{n}$ such that all their faces are riemannian, and such that they vary differentiably with respect to one or more parameters. For every $1 \leq r \leq n-2$ the following equality holds:

$$
\begin{aligned}
& \Gamma\left(\frac{r+2}{2}\right) \cdot \Gamma\left(\frac{n-r-1}{2}\right) \cdot \sum_{\operatorname{dim}(F)=r+1} \theta_{F} \cdot d \mathrm{~V}_{r+1}(F) \\
& =\Gamma\left(\frac{r}{2}\right) \cdot \Gamma\left(\frac{n-r+1}{2}\right) \cdot \sum_{\operatorname{dim}(F)=r-1} \mathrm{~V}_{r-1}(F) \cdot d \theta_{F}
\end{aligned}
$$

where the sum runs over all faces $F$ of the simplex $\Delta$ of the given dimension, $\theta_{F}$ is the algebraic measure of the polar angle at the face $F$ and $\mathrm{V}_{k}(F)$ is the ( $k$-dimensional) volume of a face $F$ of dimension $k$ of $\Delta$.

Proof. Let $F$ be a codimension $n-r-1$ face of $\Delta$. Then $F$ is an $(r+1)-$ dimensional spherical simplex, and the differential of its volume is given by the spherical Schläfli formula

$$
d \mathrm{~V}_{r+1}(F)=\frac{1}{r} \sum_{\substack{L \subset F \\ \operatorname{dim}(L)=r-1}} \mathrm{~V}_{r-1}(L) \cdot d \alpha(L, F)
$$

where the sum runs over all faces $L$ of dimension $r-1$ of $F$ and $\alpha(L, F)$ is the dihedral angle of the spherical simplex $F$ at the face $L$.

On the other hand, let $L$ be a codimension $n-r+1$ face of $\Delta$, and let $\Theta_{L}$ be the polar angle of $\Delta$ at the face $L$. Then $\Theta_{L}$ is an $(n-r)$ dimensional hyperbolic simplex, and the differential of its volume is given by the hyperbolic Schläfli formula. Since the faces of $\Theta_{L}$ coincide with the polar angles of $\Delta$ at the faces that contain $L$, we can write

$$
d \mathrm{~V}_{r}\left(\Theta_{L}\right)=\frac{-1}{n-r-1} \sum_{\substack{L \subset F \\ \operatorname{dim}(F)=r+1}} \mathrm{~V}_{n-r-2}\left(\Theta_{F}\right) \cdot d \beta\left(\Theta_{F}, \Theta_{L}\right)
$$

where the sum runs over all $(r+1)$-dimensional faces $F$ of $\Delta$ containing $L$, and $\beta\left(\Theta_{F}, \Theta_{L}\right)$ is the dihedral angle of the hyperbolic simplex $\Theta_{L}$ at the face $\Theta_{F}$. 
Now the algebraic measures of $\Theta_{F}$ and $\Theta_{L}$ are in fact $\theta_{F}= \pm \mathrm{V}_{n-r-2}\left(\Theta_{F}\right)$ and $\theta_{L}= \pm \mathrm{V}_{n-r}\left(\Theta_{L}\right)$, so we can write the last expression as follows:

$$
d \theta_{L}=\frac{-1}{n-r-1} \sum_{\substack{L \subset F \\ \operatorname{dim}(F)=r+1}} \theta_{F} \cdot \tau(L, F) d \beta\left(\Theta_{F}, \Theta_{L}\right)
$$

where $\tau(L, F)=\frac{\theta_{L} \cdot \mathrm{V}_{n-r-2}\left(\Theta_{F}\right)}{\mathrm{V}_{n-r}\left(\Theta_{L}\right) \cdot \theta_{F}} \in\{ \pm 1\}$ is a sign depending on the faces $L$ and $F$.

For the sake of clarity in the notation, let us assume that $F=\left\{x_{0} \mathbf{v}_{0}+\right.$ $\left.\cdots+x_{r+1} \mathbf{v}_{r+1} \mid x_{0} \geq 0, \ldots, x_{r+1} \geq 0\right\} \cap \mathbb{S}_{1}^{n}$, and $L=\left\{x_{0} \mathbf{v}_{0}+\cdots+\right.$ $\left.x_{r+1} \mathbf{v}_{r+1} \mid x_{0} \geq 0, \ldots, x_{r+1} \geq 0 ; x_{0}=x_{1}=0\right\} \cap \mathbb{S}_{1}^{n}$. The angle of $F$ at its $(r-1)$-dimensional face $L$ is

$$
\alpha(L, F)=\pi-\operatorname{ang}\left(\mathbf{w}_{0}^{\prime}, \mathbf{w}_{1}^{\prime}\right)
$$

where $\mathbf{w}_{0}^{\prime}, \mathbf{w}_{1}^{\prime}$ are two vectors belonging to the subspace of $\mathbb{R}_{1}^{n+1}$ spanned by $\left\{\mathbf{v}_{0}, \ldots, \mathbf{v}_{r+1}\right\}$, such that $\left\langle\mathbf{w}_{i}^{\prime}, \mathbf{v}_{j}\right\rangle=\delta_{i j}$ for $i=0,1$ and $j=0, \ldots, r+1$. Now the subspace spanned by $\left\{\mathbf{v}_{0}, \ldots, \mathbf{v}_{r+1}\right\}$ is orthogonal to the subspace spanned by $\left\{\mathbf{w}_{r+2}, \ldots, \mathbf{w}_{n}\right\}$. Hence the vectors $\mathbf{w}_{0}^{\prime}, \mathbf{w}_{1}^{\prime}$ are uniquely determined by the following conditions:

$$
\left\{\begin{array}{l}
\left\langle\mathbf{w}_{i}^{\prime}, \mathbf{v}_{j}\right\rangle=\delta_{i j} \\
\left\langle\mathbf{w}_{i}^{\prime}, \mathbf{w}_{k}\right\rangle=0
\end{array} \quad \text { for } i=0,1 ; j=0, \ldots, r+1 ; k=r+2, \ldots, n .\right.
$$

On the other hand, the polar angles of $\Delta$ at the faces $F$ and $L$ are $\Theta_{F}=$ $\left\{x_{r+2} \cdot \epsilon_{r+2} \mathbf{w}_{r+2}+\cdots+x_{n} \cdot \epsilon_{n} \mathbf{w}_{n} \mid x_{r+2} \geq 0, \ldots, x_{n} \geq 0\right\} \cap \mathbb{H}^{n}$, and $\Theta_{L}=\left\{x_{0} \cdot \epsilon_{0} \mathbf{w}_{0}+x_{1} \cdot \epsilon_{1} \mathbf{w}_{1}+\left(x_{r+2} \cdot \epsilon_{r+2} \mathbf{w}_{r+2}+\cdots+x_{n} \cdot \epsilon_{n} \mathbf{w}_{n}\right) \mid x_{0} \geq 0, x_{1} \geq\right.$ $\left.0, x_{r+2} \geq 0, \ldots, x_{n} \geq 0\right\} \cap \mathbb{H}^{n}$, respectively. Their algebraic measures are $\theta_{F}=\epsilon_{r+2} \cdot \ldots \cdot \epsilon_{n} \cdot \mathrm{V}_{n-r-2}\left(\Theta_{F}\right)$ and $\theta_{L}=\epsilon_{0} \cdot \epsilon_{1} \cdot \epsilon_{r+2} \cdots \epsilon_{n} \cdot \mathrm{V}_{n-r}\left(\Theta_{L}\right)$. Hence the sign $\tau(L, F)$ appearing in the Schläfli formula (10) is $\tau(L, F)=\epsilon_{0} \cdot \epsilon_{1}$.

The dihedral angle of the hyperbolic simplex $\Theta_{L}$ at the face $\Theta_{F}$ is

$$
\beta\left(\Theta_{F}, \Theta_{L}\right)=\pi-\operatorname{ang}\left(\mathbf{v}_{0}^{\prime}, \mathbf{v}_{1}^{\prime}\right)
$$

where $\mathbf{v}_{0}^{\prime}, \mathbf{v}_{1}^{\prime}$ are two vectors belonging to the subspace of $\mathbb{R}_{1}^{n+1}$ spanned by $\left\{\epsilon_{0} \mathbf{w}_{0}, \epsilon_{1} \mathbf{w}_{1}, \epsilon_{r+2} \mathbf{w}_{r+2}, \ldots, \epsilon_{n} \mathbf{w}_{n}\right\}$, such that $\left\langle\mathbf{v}_{i}^{\prime}, \epsilon_{j} \mathbf{w}_{j}\right\rangle=\delta_{i j}$ for $i=0,1$ and $j=0,1, r+2, \ldots, n$. Again the subspace spanned by $\left\{\epsilon_{0} \mathbf{w}_{0}, \epsilon_{1} \mathbf{w}_{1}\right.$, $\left.\epsilon_{r+2} \mathbf{w}_{r+2}, \ldots, \epsilon_{n} \mathbf{w}_{n}\right\}$ is orthogonal to the subspace spanned by $\left\{\mathbf{v}_{2}, \ldots\right.$, $\left.\mathbf{v}_{r+1}\right\}$. Hence the vectors $\mathbf{v}_{0}^{\prime}, \mathbf{v}_{1}^{\prime}$ are uniquely determined by the following conditions:

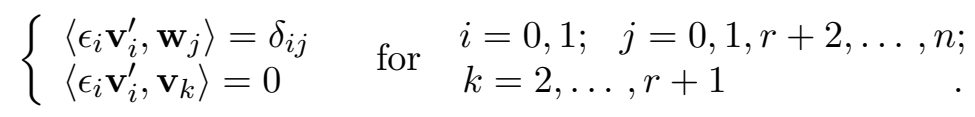

Consider the subspace $\Pi$ of $\mathbb{R}_{1}^{n+1}$ spanned by the vectors $\left\{\mathbf{v}_{2}, \ldots, \mathbf{v}_{r+1}\right.$, $\left.\mathbf{w}_{r+2}, \ldots, \mathbf{w}_{n}\right\}$. It has codimension 2 in $\mathbb{R}_{1}^{n+1}$, because it is the direct sum of the two mutually orthogonal subspaces spanned by $\left\{\mathbf{v}_{2}, \ldots, \mathbf{v}_{r+1}\right\}$ and 
$\left\{\mathbf{w}_{r+2}, \ldots, \mathbf{w}_{n}\right\}$, respectively. From conditions (11) and (12) we deduce that $\left\{\mathbf{w}_{0}^{\prime}, \mathbf{w}_{1}^{\prime}\right\}$ and $\left\{\epsilon_{0} \mathbf{v}_{0}^{\prime}, \epsilon_{1} \mathbf{v}_{1}^{\prime}\right\}$ are in fact dual basis of the plane $\Pi^{\perp}$, orthogonal to $\Pi$ in $\mathbb{R}_{1}^{n+1}$. Hence

$$
\operatorname{ang}\left(\mathbf{w}_{0}^{\prime}, \mathbf{w}_{1}^{\prime}\right)=\pi-\operatorname{ang}\left(\epsilon_{0} \mathbf{v}_{0}^{\prime}, \epsilon_{1} \mathbf{v}_{1}^{\prime}\right)
$$

and therefore

$$
\begin{aligned}
d \alpha(L, F) & =-d\left(\operatorname{ang}\left(\mathbf{w}_{0}^{\prime}, \mathbf{w}_{1}^{\prime}\right)\right) \\
& =d\left(\operatorname{ang}\left(\epsilon_{0} \mathbf{v}_{0}^{\prime}, \epsilon_{1} \mathbf{v}_{1}^{\prime}\right)\right) \\
& =\epsilon_{0} \epsilon_{1} \cdot d\left(\operatorname{ang}\left(\mathbf{v}_{0}^{\prime}, \mathbf{v}_{1}^{\prime}\right)\right) \\
& =-\tau(L, F) \cdot d \beta\left(\Theta_{F}, \Theta_{L}\right) .
\end{aligned}
$$

Applying now the Schläfli formulas (9) and (10), we get

$$
\begin{aligned}
& \sum_{\operatorname{dim}(F)=r+1} \theta_{F} \cdot d \mathrm{~V}_{r+1}(F) \\
= & \frac{1}{r} \sum_{\operatorname{dim}(F)=r+1} \sum_{\substack{L \subset F \\
\operatorname{dim}(L)=r-1}} \theta_{F} \cdot \mathrm{V}_{r-1}(L) \cdot d \alpha(L, F) \\
= & \frac{-1}{r} \sum_{\operatorname{dim}(L)=r-1} \sum_{\substack{L \subset F \\
\operatorname{dim}(F)=r+1}} \mathrm{~V}_{r-1}(L) \cdot \theta_{F} \cdot \tau(L, F) \cdot d \beta\left(\Theta_{F}, \Theta_{L}\right) \\
= & \frac{n-r-1}{r} \sum_{\operatorname{dim}(L)=r-1} \mathrm{~V}_{r-1}(L) \\
& \left.\frac{-1}{n-r-1} \sum_{\substack{L \subset F \\
\operatorname{dim}(F)=r+1}} \theta_{F} \cdot \tau(L, F) d \beta\left(\Theta_{F}, \Theta_{L}\right)\right) \\
= & \sum_{\operatorname{dim}(L)=r-1} \mathrm{~V}_{r-1}(L) \cdot d \theta_{L} .
\end{aligned}
$$

Using the properties of the gamma function we finally arrive at the desired formula.

Let us define the following constants (cf. $\left.\left[\mathbf{S a}_{1}\right]\right)$ for $0 \leq i \leq n-1$ :

$$
c_{i}=\frac{\Gamma\left(\frac{i+1}{2}\right) \cdot \Gamma\left(\frac{n-i}{2}\right)}{2 \Gamma\left(\frac{n+1}{2}\right)}=\frac{\operatorname{Vol}\left(\mathbb{S}^{n}\right)}{\operatorname{Vol}\left(\mathbb{S}^{i}\right) \cdot \operatorname{Vol}\left(\mathbb{S}^{n-1-i}\right)} .
$$

Then for $1 \leq r \leq n-2$, the equality (8) can also be written as follows:

$$
\text { (13) } \quad c_{r+1} \cdot \sum_{\operatorname{dim}(F)=r+1} \theta_{F} \cdot d \mathrm{~V}_{r+1}(F)-c_{r-1} \cdot \sum_{\operatorname{dim}(F)=r-1} \mathrm{~V}_{r-1}(F) \cdot d \theta_{F}=0
$$


Remark 3.1. In the proof of Proposition 3.1 we have never used the fact that the simplex $\Delta$ is compact. Therefore, formula (13) holds for any nonempty subset of the de Sitter sphere $\mathbb{S}_{1}^{n}$ which is the intersection of $\mathbb{S}_{1}^{n}$ with a simplicial cone of $\mathbb{R}_{1}^{n+1}$, and such that all its faces of codimension $\geq 1$ are riemannian. (For example, the polar dual $\Delta^{*}$ of a hyperbolic $n$-simplex $\Delta$, cf. §2.1.)

\section{A Gauss-Bonnet formula.}

Proposition 3.2. Let $\Delta$ be an n-simplex in the de Sitter sphere $\mathbb{S}_{1}^{n}$ such that all its faces are riemannian. Then

$$
\sum_{k=0}^{\left[\frac{n}{2}\right]}(-1)^{k} \cdot c_{2 k} \cdot \sum_{\operatorname{dim}(F)=2 k} V_{2 k}(F) \cdot \theta_{F}=0
$$

where the sum runs over all faces $F$ of $\Delta$ of even dimension, $V_{2 k}(F)$ is the volume of a face $F$ of dimension $2 k, \theta_{F}$ is the algebraic measure of the polar angle of $\Delta$ at the face $F$, and $c_{i}$ is the constant defined by

$$
\begin{array}{r}
c_{i}=\frac{\operatorname{Vol}\left(\mathbb{S}^{n}\right)}{\operatorname{Vol}\left(\mathbb{S}^{i}\right) \cdot \operatorname{Vol}\left(\mathbb{S}^{n-1-i}\right)}=\frac{\Gamma\left(\frac{i+1}{2}\right) \cdot \Gamma\left(\frac{n-i}{2}\right)}{2 \Gamma\left(\frac{n+1}{2}\right)} \\
\text { if } 0 \leq i \leq n-1 \quad \text { and } \quad c_{n}=1 .
\end{array}
$$

(We are using here the following conventions: if $k=\frac{n}{2}$, then the polar angle $\theta_{\Delta}$ at the whole simplex is taken to be equal to -1 ; if $k=\frac{n-1}{2}$, then the polar angles at the codimension 1 faces are \pm 1 (see Definition 3.1), and if $k=0$, then the volume of a vertex is always 1 .)

Remark 3.2. For even dimension $n$, the Gauss-Bonnet formula (14) gives an expression of the volume of the simplex $\Delta$ in terms of the volumes of simplices of lesser dimension:

$$
(-1)^{\frac{n}{2}} \cdot \mathrm{V}_{n}(\Delta)=\sum_{k=0}^{\frac{n}{2}-1}(-1)^{k} \cdot c_{2 k} \cdot \sum_{\operatorname{dim}(F)=2 k} \mathrm{~V}_{2 k}(F) \cdot \theta_{F} \quad \text { if } n \text { is even. }
$$

When $n$ is odd, the Gauss-Bonnet formula does not involve the volume of the simplex itself. Therefore, it cannot be used to reduce $\mathrm{V}_{n}(\Delta)$ to volumes in smaller dimensions:

$$
\sum_{k=0}^{\frac{n-1}{2}}(-1)^{k} \cdot c_{2 k} \cdot \sum_{\operatorname{dim}(F)=2 k} \mathrm{~V}_{2 k}(F) \cdot \theta_{F}=0 \quad \text { if } n \text { is odd. }
$$


Proof. We will only give the proof in the case when $n$ is even. The other case is analogous.

Let $\Delta$ be an $n$-simplex in $\mathbb{S}_{1}^{n}$ such that all its faces are riemannian, where $n$ is even. The Schläfli formula in the de Sitter sphere (Theorem 1.1) says that

$$
d \mathrm{~V}_{n}(\Delta)=\frac{1}{n-1} \sum_{F} \mathrm{~V}_{n-2}(F) d \alpha_{F}
$$

where the sum is taken over all codimension 2 faces $F$ of $\Delta$ and $\alpha_{F}$ is the dihedral angle of $\Delta$ at the face $F$. Now from the definitions of dihedral angle and polar angle it follows easily that the polar angle of $\Delta$ at the face $F$ is $\theta_{F}=-\alpha_{F}$. Considering also that $c_{n-2}=\frac{\Gamma\left(\frac{n-1}{2}\right)}{2 \Gamma\left(\frac{n+1}{2}\right)}=\frac{1}{n-1}$, we can write

$$
(-1)^{\frac{n}{2}} \cdot d \mathrm{~V}_{n}(\Delta)=(-1)^{\frac{(n-2)}{2}} \cdot c_{n-2} \cdot \sum_{\operatorname{dim}(F)=n-2} \mathrm{~V}_{n-2}(F) d \theta_{F} .
$$

On the other hand, from formula (13) we get

$$
\left\{\begin{array}{c}
(-1)^{\frac{(n-2)}{2}} \cdot c_{n-2} \cdot \sum_{\operatorname{dim}(F)=n-2} \mathrm{~V}_{n-2}(F) d \theta_{F}=(-1)^{\frac{n}{2}} \cdot d \mathrm{~V}_{n}(\Delta) \\
(-1)^{\frac{(n-2)}{2}} \cdot c_{n-2} \cdot \sum_{\operatorname{dim}(F)=n-2} \theta_{F} d \mathrm{~V}_{n-2}(F)+(-1)^{\frac{(n-4)}{2}} \cdot c_{n-4} \\
\cdot \sum_{\operatorname{dim}(F)=n-4} \mathrm{~V}_{n-4}(F) d \theta_{F}=0 \\
\vdots \\
c_{4} \cdot \sum_{\operatorname{dim}(F)=4} \theta_{F} d \mathrm{~V}_{4}(F)-c_{2} \cdot \sum_{\operatorname{dim}(F)=2} \mathrm{~V}_{2}(F) d \theta_{F}=0 \\
-c_{2} \cdot \sum_{\operatorname{dim}(F)=2} \theta_{F} d \mathrm{~V}_{2}(F)+c_{0} \cdot \sum_{\operatorname{dim}(F)=0} d \theta_{F}=0 .
\end{array}\right.
$$

Adding up all these equalities and integrating, we obtain

$$
(-1)^{\frac{n}{2}} \cdot \mathrm{V}_{n}(\Delta)=\sum_{k=0}^{\frac{n}{2}-1}(-1)^{k} \cdot c_{2 k} \cdot \sum_{\operatorname{dim}(F)=2 k} \mathrm{~V}_{2 k}(F) \cdot \theta_{F}+\text { constant. }
$$

The constant can be found to be zero by considering the limiting case as the polar hyperbolic simplex $\Delta^{*}$ degenerates to a point. Then all polar angles $\theta_{F} \rightarrow 0$, and the simplex $\Delta$ degenerates into a subset of a hyperplane, so $\mathrm{V}_{n}(\Delta) \rightarrow 0$.

Remark 3.3. I have called formula (14) a Gauss-Bonnet formula because of its analogy to the generalized Gauss-Bonnet formulas for convex polyhedra in non-Euclidean space of constant curvature $\kappa= \pm 1$ (cf. [Saa $]$, [AVS]). 
There is, however, an essential difference in that the right-hand side of Equation (14) is zero. Therefore, if we tried to extend the formula (14) to polyhedra in $\mathbb{S}_{1}^{n}$ which admit a triangulation by simplices with riemannian faces, the Euler characteristic of the polyhedron would never appear.

Remark 3.4. The same procedure used in the proofs of Propositions 3.1 and 3.2 can also be applied in the spherical and hyperbolic case to prove the above mentioned Gauss-Bonnet formulas for spherical and hyperbolic simplices.

\section{Dual volume of a hyperbolic $n$-simplex. (A generalization of Santaló's formula.)}

Let $\Delta=\left\{x_{0} \mathbf{v}_{0}+\cdots+x_{n} \mathbf{v}_{n} \mid x_{0} \geq 0, \ldots, x_{n} \geq 0\right\} \cap \mathbb{H}^{n}$ be a hyperbolic $n$-simplex, and let $\left\{\mathbf{w}_{0}, \ldots, \mathbf{w}_{n}\right\}$ be the dual basis $\left\{\mathbf{v}_{0}, \ldots, \mathbf{v}_{n}\right\}$. Consider the polar dual of $\Delta, \Delta^{*}=\left\{x_{0} \mathbf{w}_{0}+\cdots+x_{n} \mathbf{w}_{n} \mid x_{0} \geq 0, \ldots, x_{n} \geq 0\right\} \cap \mathbb{S}_{1}^{n}$, and the complementary dual, $\Delta^{P}=\left\{x_{0} \mathbf{w}_{0}+\cdots+x_{n} \mathbf{w}_{n} \mid x_{0} \geq 0\right.$ and $x_{i} \leq$ 0 for some $i \in\{1, \ldots, n\}\} \cap \mathbb{S}_{1}^{n}$. We saw in Section 2.1 that the dual volume of $\Delta$ (i.e., the measure of the set of hyperbolic hyperplanes intersecting it) is equal to the volume of its complementary dual $\Delta^{P}$. Now we are going to find a relation between this dual volume $V_{n}\left(\Delta^{P}\right)$ and the volumes of all odd-dimensional faces of the hyperbolic simplex $\Delta$ and the polar angles at those faces.

Definition 4.1. For $0 \leq k \leq n-1$, we will associate to each face of codimension $k+1$ of the simplex $\Delta$,

$$
F=\left\{x_{0} \mathbf{v}_{0}+\cdots+x_{n} \mathbf{v}_{n} \mid x_{0} \geq 0, \ldots, x_{n} \geq 0 ; x_{i_{1}}=\cdots=x_{i_{k}}=0\right\} \cap \mathbb{H}^{n}
$$

the following $k$-dimensional face of the polar dual $\Delta^{*}$ :

$$
F^{*}=\left\{x_{i_{1}} \mathbf{v}_{i_{1}}+\cdots+x_{i_{k}} \mathbf{v}_{i_{k}} \mid x_{i_{1}} \geq 0, \ldots, x_{i_{k}} \geq 0\right\} \cap \mathbb{S}_{1}^{n} .
$$

We will call $F$ and $F^{*}$ polar faces. We define the polar angle $\theta_{F}$ of the hyperbolic simplex $\Delta$ at the face $F$ as the volume of its polar face $F^{*}$ (which is a $k$-dimensional spherical simplex).

Remark 4.1. If $F$ is a codimension 2 face of $\Delta$ and $\alpha_{F}$ is the dihedral angle of $\Delta$ at $F$, then the polar angle at $F$ is $\theta_{F}=\pi-\alpha_{F}$.

Proposition 4.1. Let $\Delta$ be a hyperbolic n-simplex. Then its dual volume is

$$
\mathrm{V}_{n}\left(\Delta^{P}\right)=\sum_{k=0}^{\left[\frac{n-1}{2}\right]}(-1)^{k} \cdot c_{2 k+1} \cdot \sum_{\operatorname{dim}(F)=2 k+1} V_{2 k+1}(F) \cdot \theta_{F}
$$

where the sum extends over all odd-dimensional faces $F$ of $\Delta, V_{2 k+1}(F)$ is the volume of a face $F$ of dimension $2 k+1, \theta_{F}$ is the polar angle of $\Delta$ at 
the face $F$, and $c_{i}$ is the constant defined by

$$
\begin{aligned}
c_{i} & =\frac{\operatorname{Vol}\left(\mathbb{S}^{n}\right)}{\operatorname{Vol}\left(\mathbb{S}^{i}\right) \cdot \operatorname{Vol}\left(\mathbb{S}^{n-1-i}\right)} \\
& =\frac{\Gamma\left(\frac{i+1}{2}\right) \cdot \Gamma\left(\frac{n-i}{2}\right)}{2 \Gamma\left(\frac{n+1}{2}\right)} \quad \text { if } 0 \leq i \leq n-1 \quad \text { and } \quad c_{n}=1 .
\end{aligned}
$$

(We are using here the following conventions: if $k=\frac{n-1}{2}$, then the polar angle $\theta_{\Delta}$ at the whole simplex is taken to be equal to 1 ; if $k=\frac{n-2}{2}$, then the polar angles at the codimension 1 faces are 1 , and if $k=0$, then the volume of a vertex is always 1.)

Remark 4.2. For odd dimension $n$, formula (15) relates the volume of the $n$ - simplex $\Delta$ with its dual volume:

$$
\begin{aligned}
& \mathrm{V}_{n}\left(\Delta^{P}\right)+(-1)^{\frac{n+1}{2}} \cdot \mathrm{V}_{n}(\Delta) \\
& =\sum_{k=0}^{\frac{n-3}{2}}(-1)^{k} \cdot c_{2 k+1} \cdot \sum_{\operatorname{dim}(F)=2 k+1} \mathrm{~V}_{2 k+1}(F) \cdot \theta_{F} \quad \text { if } n \text { is odd. }
\end{aligned}
$$

In particular, in dimension 3 we obtain again Santaló's formula for hyperbolic tetrahedra.

When $n$ is even, formula (15) relates the dual volume of the $n$-simplex $\Delta$ with its "surface area" $S_{n-1}(\Delta)$, i.e., with the sum of the volumes of all its faces of codimension 1 :

$$
\begin{aligned}
& \mathrm{V}_{n}\left(\Delta^{P}\right)+(-1)^{\frac{n}{2}} \cdot c_{n-1} \cdot \mathrm{S}_{n-1}(\Delta) \\
& =\sum_{k=0}^{\frac{n-4}{2}}(-1)^{k} \cdot c_{2 k+1} \cdot \sum_{\operatorname{dim}(F)=2 k+1} \mathrm{~V}_{2 k+1}(F) \cdot \theta_{F} \quad \text { if } n \text { is even. }
\end{aligned}
$$

In particular, in dimension 2 this says that the measure of the set of all lines intersecting a planar hyperbolic triangle, is equal to the perimeter of the triangle. In dimension 4 , the expression is a little more complicated:

$$
3 \mathrm{~V}_{4}\left(\Delta^{P}\right)+2 \mathrm{~S}_{3}(\Delta)=\sum_{\operatorname{dim}(F)=1} \mathrm{~V}_{1}(F) \cdot \theta_{F}
$$

Proof. We will do it only when $n$ is odd, since the other case is analogous.

Let us apply the formulas (13) of Section 3 to the polar dual $\Delta^{*}$ of $\Delta$ (cf. Remark 3.1). Since the constants $c_{i}$ satisfy the relation $c_{i}=c_{n-i-1}$, we 
have that for $2 \leq r \leq n-1$,

$$
\begin{aligned}
& c_{r-2} \cdot \sum_{\operatorname{dim}\left(F^{*}\right)=n-r+1} \theta_{F^{*}} \cdot d \mathrm{~V}_{n-r+1}\left(F^{*}\right) \\
& -c_{r} \cdot \sum_{\operatorname{dim}\left(F^{*}\right)=n-r-1} \mathrm{~V}_{n-r-1}\left(F^{*}\right) \cdot d \theta_{F^{*}}=0
\end{aligned}
$$

where the sums extend to all faces $F^{*}$ of the polar dual $\Delta^{*}$ of the given dimension, $\theta_{F^{*}}$ is the algebraic measure of the polar angle of $\Delta^{*}$ at the face $F^{*}$, and $\mathrm{V}_{k}\left(F^{*}\right)$ is the ( $k$-dimensional) volume of a $k$-dimensional face $F^{*}$ of $\Delta^{*}$.

Now the dual basis $\left\{\mathbf{v}_{0}, \ldots, \mathbf{v}_{n}\right\}$ of $\left\{\mathbf{w}_{0}, \ldots, \mathbf{w}_{n}\right\}$ is formed by timelike vectors contained in the upper timecone. Therefore, the algebraic measure of the polar angle of $\Delta^{*}$ at the face $F^{*}$ coincides with the volume of the polar face $F$ of $\Delta$ (cf. Definition 3.1). Hence if $F \subset \Delta$ and $F^{*} \subset \Delta^{*}$ are polar faces of dimensions $r$ and $n-r-1$, respectively, then

$$
\mathrm{V}_{r}(F)=\theta_{F^{*}} \quad \text { and } \quad \mathrm{V}_{n-r-1}\left(F^{*}\right)=\theta_{F} .
$$

Therefore, for $1 \leq r \leq n-2$ the equality (16) can be written in terms of quantities associated only to the hyperbolic simplex $\Delta$ itself, as follows:

$$
c_{r-2} \cdot \sum_{\operatorname{dim}(F)=r-2} \mathrm{~V}_{r-2}(F) \cdot d \theta_{F}-c_{r} \cdot \sum_{\operatorname{dim}(F)=r} \theta_{F} \cdot d \mathrm{~V}_{r}(F)=0
$$

where the sums run over all faces $F$ of $\Delta$ of the given dimension, $\theta_{F}$ is the polar angle of $\Delta$ at the face $F$, and $\mathrm{V}_{k}(F)$ is the ( $k$-dimensional) volume of a face $F$ of dimension $k$ of $\Delta$.

On the other hand, considering the Remark 4.1 and the fact that $c_{1}=$ $c_{n-2}=\frac{1}{n-1}$, we know from the hyperbolic Schläfli formula that

$$
d \mathrm{~V}_{n}(\Delta)=c_{n-2} \cdot \sum_{\operatorname{dim}(F)=n-2} \mathrm{~V}_{n-2}(F) d \theta_{F}
$$

and from the Schläfli formula in the de Sitter sphere, that

$$
d \mathrm{~V}_{n}\left(\Delta^{P}\right)=c_{1} \cdot \sum_{\operatorname{dim}\left(F^{*}\right)=n-2} \mathrm{~V}_{n-2}\left(F^{*}\right) d \alpha_{F^{*}}
$$

where we sum over all codimension 2 faces $F^{*}$ of $\Delta^{*}$, and $\alpha_{F^{*}}$ is the dihedral angle of $\Delta^{P}$ at the face $F^{*}$. But $\alpha_{F^{*}}=\mathrm{V}_{1}(F)$ and $\mathrm{V}_{n-2}\left(F^{*}\right)=\theta_{F}$, where $F$ is the polar face of $F^{*}$. Hence

$$
d \mathrm{~V}_{n}\left(\Delta^{P}\right)=c_{1} \cdot \sum_{\operatorname{dim}(F)=1} \theta_{F} d \mathrm{~V}_{1}(F) .
$$


Let us combine formulas (17), (18) and (19):

$$
\left\{\begin{array}{c}
c_{1} \cdot \sum_{\operatorname{dim}(F)=1} \theta_{F} d \mathrm{~V}_{1}(F)=d \mathrm{~V}_{n}\left(\Delta^{P}\right) \\
c_{1} \cdot \sum_{\operatorname{dim}(F)=1} \mathrm{~V}_{1}(F) d \theta_{F}-c_{3} \cdot \sum_{\operatorname{dim}(F)=3} \theta_{F} d \mathrm{~V}_{3}(F)=0 \\
\vdots \\
(-1)^{\frac{(n-5)}{2}} \cdot c_{n-4} \cdot \sum_{\operatorname{dim}(F)=n-4} \mathrm{~V}_{n-4}(F) d \theta_{F}+(-1)^{\frac{(n-3)}{2}} \cdot c_{n-2} \\
\cdot \sum_{\operatorname{dim}(F)=n-2} \theta_{F} d \mathrm{~V}_{n-2}(F)=0 \\
(-1)^{\frac{(n-3)}{2}} \cdot c_{n-2} \cdot \sum_{\operatorname{dim}(F)=n-2} \mathrm{~V}_{n-2}(F) d \theta_{F}=(-1)^{\frac{(n-3)}{2}} \cdot d \mathrm{~V}_{n}(\Delta) .
\end{array}\right.
$$

Adding up all these equalities and integrating we obtain

$$
\begin{aligned}
& \mathrm{V}_{n}\left(\Delta^{P}\right)+(-1)^{\frac{(n-3)}{2}} \cdot \mathrm{V}_{n}(\Delta) \\
& =\sum_{k=0}^{\frac{n-3}{2}}(-1)^{k} \cdot c_{2 k+1} \cdot \sum_{\operatorname{dim}(F)=2 k+1} \mathrm{~V}_{2 k+1}(F) \cdot \theta_{F}+\text { constant. }
\end{aligned}
$$

Again we see that the constant is zero by considering the limiting case as $\Delta$ degenerates to a point (and $\Delta^{P}$ degenerates to a hyperplane in $\mathbb{S}_{1}^{n}$ ).

\section{References}

[AVS] D.V. Alekseevskij, E.B. Vinberg and A.S. Solodovnikov, Geometry of spaces of constant curvature, Geometry II (E.B. Vinberg, ed.), Encyclopedia of Mathematical Sciences, Vol. 29, Springer Verlag, (1993), 1-138.

[Bo] F. Bonahon, A Schläfli-type formula for convex cores of hyperbolic 3-manifolds, J. Diff. Geom., 50(1) (1998), 25-58.

$\left[\mathrm{HLM}_{1}\right]$ H.M. Hilden, M.T. Lozano and J.M. Montesinos, On a remarkable polyhedron geometrizing the figure eight knot cone manifolds, J. Math. Sci. Univ. Tokyo, 2 (1995), 501-561.

$\left[\mathrm{HLM}_{2}\right] \_$, On volumes and Chern-Simons invariants of geometric 3-manifolds, J. Math. Sci. Univ. Tokyo, 3 (1996), 723-744.

$\left[\mathrm{HLM}_{3}\right] \ldots$, Volumes and Chern-Simons invariants of cyclic coverings over rational knots, Proc. of the 37th Taniguchi Symposium on Topology and Teichmüller spaces, Finland, July 1995 (S. Kojima et al., eds.), World Scientific Publishing Co., (1996), 31-55.

[Ho] C. Hodgson, Degeneration and regeneration of geometric structures on 3manifolds, Ph.D. thesis, Princeton University, 1986.

[HR] C.D. Hodgson and I. Rivin, A characterization of compact convex polyhedra in hyperbolic 3-space, Invent. Math., 111 (1993), 77-111. 
[Kel] R. Kellerhals, On the volume of hyperbolic polyhedra, Math. Ann., 285 (1989), 541-569.

[Kne] H. Kneser, Der Simplexinhalt in der nichteuklidischen Geometrie, Deutsche Math., 1 (1936), 337-340.

[Mil] J. Milnor, The Schläfli differential equality, Collected papers, Vol. 1, Publish or Perish, 1994.

[Mo] J.M. Montesinos, Schläfli formula with integral geometry? (unpublished manuscript contained in a letter to Professor L. Santaló of 18-6-93).

[O'N] B. O'Neill, Semi-Riemannian Geometry with applications to Relativity, Academic Press, 1983.

[Ri] I. Rivin, On geometry of convex polyhedra in hyperbolic 3-space, Ph.D. thesis, Princeton, 1986.

[RS] I. Rivin and J-M. Schlenker, The Schläfli formula in Einstein manifolds with boundary, Electron. Res. Announc. Am. Math. Soc., 5(3) (1999), 18-23.

[Sa $\left.{ }_{1}\right] \quad$ L. Santaló, Sobre la fórmula de Gauss-Bonnet para poliedros en espacios de curvatura constante, Revista de la Union Matemática Argentina, XX (1960), 79-91.

$\left[\mathrm{Sa}_{2}\right]$, Integral Geometry and Geometric Probability, Encyclopedia of Mathematics and its applications, Vol. 1, Addison-Wesley, 1976.

[Schlä] L. Schläfli, On the multiple integral $\int^{n} d x d y \ldots d z$, whose limits are $p_{1}=a_{1} x+$ $b_{1} y+\cdots+h_{1} z>0, p_{2}>0, \cdots, p_{n}>0$, and $x^{2}+y^{2}+\cdots+z^{2}<1$, Quart. J. Pure Appl. Math., 2 (1858), 69-301.

[Schle 1 ] J-M. Schlenker, Polyèdres dans les espaces de Sitter-hyperboliques, preprint, 1996.

[Schle $\left.{ }_{2}\right] \ldots$, Métriques sur les polyèdres hyperboliques convexes, Journal of Differential Geometry, 48 (1998), 323-405.

[S-S] J-M. Schlenker and R. Souam, Higher Schläfli formulas and applications, preprint, 1999.

[Su] E. Suárez-Peiró, Poliedros de Dirichlet de 3-variedades cónicas y sus deformaciones, Ph.D. thesis, Universidad Complutense de Madrid, 1998.

Received July 17, 1997 and revised March 21, 1999.

Mathematisches Institut Der Universität TüBIngen

Auf Der Morgenstelle 10

72076 TÜBINGEN

Germany

E-mail address: eva@riemann.mathematik.uni-tuebingen.de 